Sädhanā, Vol. 19, Part 5, October 1994, pp. 619-647. (C) Printed in India.

\title{
The differentially heated cavity
}

\author{
S PAOLUCCI
}

Aerospace and Mechanical Engineering Department, University of Notre Dame, IN 46556-5637, USA

\begin{abstract}
This review discusses recent work dealing with natural convection flow in a differentially heated cavity. The emphasis is placed primarily on work dealing with the non-Boussinesq regime, transitional flow, and turbulent flow. Direction for future work in areas where additional effort is required is also provided.
\end{abstract}

Keywords. Natural convection; non-Boussinesq; transition; turbulence.

\section{Introduction}

Flow in rectangular enclosures are encountered in a variety of industrial applications. Because of their importance, such flows have been the subject of research for many decades. An attempt at a complete review and history of this subject is a formidable task which I will not attempt here. Instead, I will discuss the more recent results that have some technological importance and also reflect my current interest.

My discussion will deal exclusively with the study of natural convection flow within a vertical rectangular enclosure resulting from lateral heating in the presence of gravity. This is a prototypical problem that is relevant to many applications, such as thermal insulation of buildings, solar energy storage, crystal growth, and nuclear reactor core isolation. In many of these applications, direct modelling of the physical processes is rather complex. As a result, natural convection flow in this idealized configuration offers the opportunity for researchers to understand the more fundamental aspects of the resulting flows. In point of fact, this prototypical problem is considered as a benchmark in evaluating computational methods in the laminar (low Rayleigh number) regime. More importantly, this problem offers the opportunity to fully understand the transition mechanisms and gain substantial insight into natural convection turbulence. Since the flow is fully enclosed, boundary conditions are well defined. Ambient turbulent fluid fluctuations, that are difficult in general to characterize, do not enter in studies of flow transition and turbulence within the cavity. Furthermore, because the flow in fully bounded, no artificial (and generally incorrect) boundary conditions need be introduced in computational studies.

Excellent reviews of the earlier works on this problem are given by Ostrach (1972, pp. 161-227, 1982, pp. 365-79), Catton (1978, pp. 13-30), and Yang (1987, pp. 13.1-13.51, 1988). After discussing simplifying hypotheses that are often used in analyses of the problem, we will briefly review some of the earlier works, but more 
emphasis is placed on more recent works dealing with non-Boussinesq effects, and studies that have shed some light on instabilities, transition, and turbulence within the cavity.

\section{Problem definition}

Consider a three-dimensional enclosure of width $L$, height $H$, and depth $D$ containing a fluid as shown in figure 1 . The fluid is initially quiescent and at a uniform temperature $T_{0}^{*}$ and pressure $p_{0}^{*}$. The walls of the container are initially at the same temperature $T_{0}^{*}$. The $x^{*}$-coordinate is fixed on the left wall, and the $y^{*}$-coordinate is positive in the upwards direction. At times larger than zero the two vertical walls located at $x^{*}=0, L$ are maintained at temperatures $T_{h}^{*}$ and $T_{c}^{*}$ respectively, where $\Delta T \equiv T_{h}^{*}-$ $T_{c}^{*}>0$. Asterisk superscripts denote dimensional quantities.

We keep assumptions to a minimum at this stage and non-dimensionalize the problem with reference quantities for length, velocity, temperature, and thermodynamic pressure using the width $L$, the viscous diffusion speed $u_{r}=\alpha_{r} / L$, the average temperature $T_{r}=\left(T_{h}^{*}+T_{c}^{*}\right) / 2$, and the initial pressure $p_{r}=p_{0}^{*}$, respectively, as follows:

$$
\begin{aligned}
& x_{i}^{*}=L x_{i}, \quad t^{*}=\left(L / u_{r}\right) t, \quad u_{i}^{*}=u_{r} u_{i}, \quad \Pi^{*}=\rho_{r} u_{r}^{2} \Pi, \\
& \rho^{*}=\rho_{r} \rho, \quad T^{*}=T_{r} T, \quad \bar{p}^{*}=p_{r} \bar{p}, \quad c_{p}^{*}=c_{p r} c_{p}, \\
& \beta^{*}=\beta_{r} \beta, \quad \mu^{*}=\mu_{r} \mu, \quad \lambda^{*}=\mu_{r} \lambda_{,} \quad k^{*}=k_{r} k,
\end{aligned}
$$

where we have introduced a number of fluid properties, all of which are evaluated at the reference temperature and thermodynamic pressure.

The resulting dimensionless governing equations, valid under low Mach number conditions (generalized from those given by Paolucci 1982), but allowing for arbitrary density variations, are given as follows:

$$
\begin{aligned}
& \partial \rho / \partial t+\partial \rho u_{i} / \partial x_{i}=0 \\
& \frac{\partial \rho u_{i}}{\partial t}+\frac{\partial \rho u_{j} u_{i}}{\partial x_{j}}=-\frac{\partial \Pi}{\partial x_{i}}+\frac{\operatorname{Ra} \operatorname{Pr}}{2 \varepsilon} \rho n_{i}+\operatorname{Pr} \frac{\partial \tau_{i j}}{\partial x_{j}}
\end{aligned}
$$

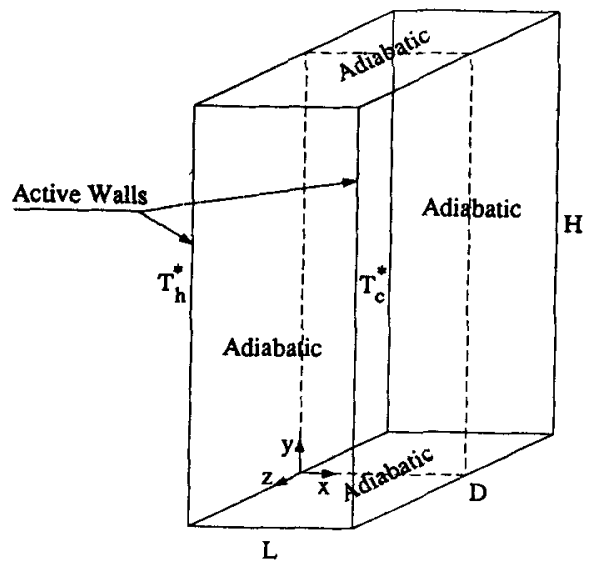

Figure 1. Schematic view of the cavity. 


$$
\begin{aligned}
& \rho c_{p}\left(\frac{\partial T}{\partial t}+u_{j} \frac{\partial T}{\partial x_{j}}\right)-\Gamma \beta T \frac{\mathrm{d} \bar{p}}{\mathrm{~d} t}=\frac{\partial}{\partial x_{j}}\left(k \frac{\partial T}{\partial x_{j}}\right), \\
& \rho=\rho(\bar{p}, T),
\end{aligned}
$$

where $u_{i}=(u, v, w)$ are velocity components in the $x_{i}=(x, y, z)$ directions respectively, $\Pi$ is the reduced pressure which accounts for the hydrostatic and hydrodynamic effect, $n_{i}=(0,-1,0)$ is the unit vector in the direction of gravity, and $\tau_{i j}$ is the viscous stress tensor given by

$$
\tau_{i j}=\mu\left(\frac{\partial u_{i}}{\partial x_{j}}+\frac{\partial u_{j}}{\partial x_{i}}\right)+\lambda \delta_{i j} \frac{\partial u_{k}}{\partial x_{k}},
$$

where $\lambda$ is the coefficient of bulk viscosity, and $\delta_{i j}$ is the Kronecker delta.

The initial and boundary conditions expressed in dimensionless form are:

$$
\begin{aligned}
& u_{i}=0, T=1 \text { at } t=0, \\
& u_{i}=0, T=1+\varepsilon \text { at } x=0 \text { and } u_{i}=0, T=1-\varepsilon \text { at } x=1, \\
& u_{i}=0, \partial T / \partial y=0 \text { at } y=0, A_{H}, \text { and } u_{i}=0, \partial T / \partial z=0 \text { at } z= \pm A_{D} / 2 .
\end{aligned}
$$

The spatially uniform pressure $\bar{p}=\bar{p}(t)$ appearing in the energy equation and the equation of state accounts for the change of static pressure with time. The separation of pressure components is essential in removing acoustic waves from the equations, however this splitting introduces $\bar{p}$ as an extra unknown. Now the general equation of state can be rewritten more explicitly as

$$
\rho=\exp \left(-\int_{1}^{T} \beta \mathrm{d} T^{\prime}+\int_{1}^{\bar{p}} \kappa \mathrm{d} p^{\prime}\right),
$$

where $\beta=-(\partial \rho / \partial T)_{\bar{p}} / \rho$ is the coefficient of volume expansion, and $\kappa=(\partial \rho / \partial \bar{p})_{T} / \rho$ is the isothermal compressibility coefficient. Using the global mass conservation statement

$$
\frac{\mathrm{d}}{\mathrm{d} t} \int_{V} \rho \mathrm{d} V=0
$$

in conjunction with local continuity and energy equations, we obtain the following differential-integral equation for the static pressure,

$$
\begin{aligned}
\frac{\mathrm{d} \ddot{p}}{\mathrm{~d} t}=\left\{\int_{S} k \frac{\partial T}{\partial x_{j}} \mathrm{~d} S_{j}+\int_{V} u_{i} \frac{\partial}{\partial x_{i}}\left[\rho c_{p} /\left(\beta-\int_{1}^{\bar{p}} \frac{\partial \kappa}{\partial T} \mathrm{~d} \bar{p}^{\prime}\right)\right] \mathrm{d} V\right\} \div \\
\quad\left\{\int_{V}\left[\rho c_{p}\left(\kappa-\int_{1}^{T} \frac{\partial \beta}{\partial \bar{p}} \mathrm{~d} T^{\prime}\right) /\left(\beta-\int_{1}^{\bar{p}} \frac{\partial \kappa}{\partial T} \mathrm{~d} \bar{p}^{\prime}\right)-\Gamma\right] \mathrm{d} V\right\},
\end{aligned}
$$

where $S$ and $V$ are the surface area and volume of the cavity. This equation is complemented by the initial condition

$$
\bar{p}=1 \quad \text { at } \quad t=0 .
$$


The independent dimensionless parameters appearing in the problem are:

$$
\begin{aligned}
\mathrm{Ra} & =\beta_{r} g \Delta T L^{3} / v_{r} \alpha_{r}, \quad \operatorname{Pr}=v_{r} / \alpha_{r}, \quad \varepsilon=\frac{1}{2} \beta_{r} \Delta T, \\
\Gamma & =\left(1 / \sigma_{r} \mathrm{~T}_{r}\right)\left(\left(\gamma_{r}-1\right) / \gamma_{r}\right), \quad A_{H}=H / L, \quad A_{D}=D / L .
\end{aligned}
$$

In the above definitions and non-dimensionalizations $\rho$ is the density; $\alpha=k / \rho c_{p}$ and $v=\mu / \rho$ are the kinematic viscosity and thermal diffusivity, while $\mu$ and $k$ are the dynamic viscosity and thermal conductivity; $c_{p}, c_{v}$ and $\gamma$ are the specific heats at constant pressure and volume and their ratio; $\sigma=(\partial \bar{p} / \partial T)_{\rho} / \bar{p}$ is the coefficient of tension; and lastly $g$ is the magnitude of the gravitational field.

We emphasize the fact that (2), (3), and (7) are applicable to the natural convection flow of any fluid within a fully enclosed cavity. No assumptions regarding property variations are made. Solutions of these equations account for all non-Boussinesq effects in natural convection. Most non-Boussinesq results to date are for air, whose flow may accurately be described by using the Stokes assumption $\lambda=-\frac{2}{3} \mu$ and the thermodynamic properties by the calorically perfect gas assumptions $c_{p}=1, \beta=1 / T$, $\kappa=1 / \bar{p}$, and $\sigma=1 / T$. Subsequently, we have $\Gamma=\left(\gamma_{r}-1\right) / \gamma_{r}$, and (5) and (7) simplify to

$$
\begin{aligned}
& \rho=\bar{p} / T \\
& \frac{\mathrm{d} \bar{p}}{\mathrm{~d} t}=\frac{\gamma_{r}}{V} \int_{S} k \frac{\partial T}{\partial x_{j}} \mathrm{~d} S_{j} .
\end{aligned}
$$

In addition, for air $\gamma_{r}=7 / 5, \operatorname{Pr}=0.71$, and the transport properties are accurately approximated by the Sutherland law models

$$
\mu=T^{3 / 2}\left(\frac{1+S_{\mu}}{T+S_{\mu}}\right), \quad k=T^{3 / 2}\left(\frac{1+S_{k}}{T+S_{k}}\right),
$$

where using $T_{r}=300 \mathrm{~K}$ and normal pressure, the dimensionless Sutherland constants are $S_{\mu}=S_{\mu}^{*} / T_{r}=0.368$ and $S_{k}=S_{k}^{*} / T_{r}=0.648$ (see White 1974). From the definition of $\varepsilon$ we note than $0<\varepsilon<1$ corresponds to the temperature difference range of $0<\Delta T<\infty$. Obviously the range of validity of the Sutherland law is considerably less. As a point of reference, note that $\varepsilon=0.6$ corresponds to $T_{h}^{*}=480 \mathrm{~K}$ and $T_{c}^{*}=120 \mathrm{~K}$ for $T_{r}=300 \mathrm{~K}$, and represents a practical upper limit on the validity of the results for air resulting from increasing errors in the Sutherland law conductivity at the cold wall (see Chenoweth \& Paolucci 1986).

It was shown by Paolucci (1982) that in the limit $\varepsilon \ll 1$, we have $\bar{p}=1, \mathrm{~d} \bar{p} / \mathrm{d} t=0$, and subtracting out the hydrostatic components, (2), (3), and (5) reduce to the classical Boussinesq equations

$$
\begin{aligned}
& \frac{\partial u_{i}}{\partial x_{i}}=0 \\
& \frac{\partial u_{i}}{\partial t}+u_{j} \frac{\partial u_{i}}{\partial x_{j}}=-\frac{\partial \Pi_{d}}{\partial x_{i}}-\operatorname{Ra} \operatorname{Pr} \theta n_{i}+\operatorname{Pr} \frac{\partial^{2} u_{i}}{\partial x_{j} \partial x_{j}}, \\
& \frac{\partial \theta}{\partial t}+u_{j} \frac{\partial \theta}{\partial x_{j}}=\frac{\partial^{2} \theta}{\partial x_{j} \partial x_{j}},
\end{aligned}
$$

where $\Pi_{d}$ is the dynamic component of pressure and $\theta=(T-1) / 2 E$. It is emphasized 
that the Boussinesq equations will only yield relatively accurate solutions only for small temperature differences. For example, Gray \& Giorgini (1976) show that the maximum temperature differences for air and water for which these equations are applicable are $28.6^{\circ}$ and $1.25^{\circ} \mathrm{C}$ respectively.

Solutions for the Boussinesq equations have been obtained for a large range of Prandtl numbers and primarily in a two-dimensional cavity where $A_{D} \rightarrow \infty$. Furthermore, some solutions have been obtained using perfectly conducting top and bottom boundaries so that the thermal conditions at $y=0, A_{H}$ in (4) are replaced by

$$
T=1+\varepsilon(1-2 x) \text {. }
$$

The problem studied here is perhaps the simplest one possible involving convection heat transfer between two isothermal boundaries; in spite of this apparent simplicity, the physical nature of the resulting flow regions, even at steady-state, can be an incredibly complex function of the controlling independent dimensionless parameters.

\section{Numerical techniques}

The Boussinesq equations have been solved in both streamfunction-vorticity and primitive variable formulations. Since most of the recent results deal with non-Boussinesq effects, and since any computational scheme that one hopes to apply to transition and turbulence studies must be easily extendable to three-dimensions, most of the results that will be discussed have been obtained using a primitive variable formulation.

The most accurate simulations to date have used a primitive variable formulation in conjunction with either a pseudo-spectral or a control-volume spatial approximation combined with a finite-difference time integration.

The principle of pseudo-spectral methods is the expansion of a variable in a finite series of orthogonal trial solutions. For the present problem where we have rigid walls one may use Chebysher polynomials. In the pseudo-spectral method the Chebyshev polynomials are evaluated at specific spatial locations called the GaussLobatto points. The choice of these collocation points simplifies the computation of the nonlinear terms and allows the use of Fast Fourier Transforms. The main advantage of pseudo-spectral methods is their accurate approximation of sufficiently smooth functions. However, they are restricted to simple geometries, the grid refinement distribution in the boundary layers is only trigonometric, and the construction of an efficient code is quite complicated.

Therefore, finite-difference or finite-volume methods are preferred by most researchers. However, their application must meet strict requirements. The scheme must be able to resolve accurately all scales of motion down to the size of a few grid cells. Moreover, there must be none or little numerical diffusion. These requirements rule out standard upwind schemes, which are first order and have strong numerical diffusion. Therefore, numerical schemes with at least second-order accuracy are mandatory. These schemes are usually applied on a staggered grid located on or within a finite control volume. The velocity components are defined at the centre of the sides whose normals point in the corresponding component directions, while scalar quantities are defined at the centre of the volume. For the finite difference formulation of the nonlinear convective terms one should adopt an energy conserving 
scheme to avoid nonlinear instability (see Arakawa 1966, and Piacsek \& Williams 1970). The time advancement is frequently calculated by either the Euler or the Adams-Bashforth method.

To enforce the condition of incompressibility in the Boussinesq limit, the continuity equation is reformulated in the form of a Poisson equation for the dynamic pressure (see Peyret \& Taylor 1983),

$$
\frac{\partial^{2} \Pi}{\partial x_{i} \partial x_{i}}=\frac{\partial R_{i}}{\partial x_{i}}-\frac{\partial}{\partial t}\left(\frac{\partial u_{i}}{\partial x_{i}}\right)
$$

in which $R_{i}$ contains the contributions of the nonlinear terms. To calculate the pressure at time step $n$ the last term on the right-hand side of (15) is approximated by

$$
\frac{\partial}{\partial t}\left(\frac{\partial u_{i}}{\partial x_{i}}\right) \approx \frac{1}{\Delta t}\left[\left(\frac{\partial u_{i}}{\partial x_{i}}\right)^{n+1}-\left(\frac{\partial u_{i}}{\partial x_{i}}\right)^{n}\right]
$$

The first term on the right-hand side is set equal to zero in agreement with continuity. The second term is kept as a correction in order to force the solution to a divergencefree flow field (see Deardorff 1973, pp. 271-311).

For details of analogous algorithms applied to the non-Boussinesq equations the reader is referred to Chenoweth \& Paolucci (1986) for the control volume formulation and to Le Quéré et al (1992) for the pseudo-spectral formulation.

Next we consider the boundary conditions. On the fixed walls one must use the no-slip condition for the velocity and the appropriate temperature conditions. In solving the discretized form of the Poisson equation, knowledge of the pressure gradient normal to the walls is required. A simple and popular choice is to set this gradient to zero similar to boundary layer flows. For physical reasons no boundary conditions for the pressure ought to be prescribed at the walls. A zero gradient normal to the walls, however, is not a bad approximation as long as the Rayleigh number is high so that boundary layers exist, and as long as the boundary layers are neither separated nor leaving the surface. Such is not the case in all wall regions of the cavity. A consistent and correct solution for the pressure gradient is obtained by evaluating the momentum equations at the walls.

In a direct simulation of the transition or turbulent flow, the flow field must remain completely resolved up to the wall. This sets important restrictions on the grid spacing. Therefore, one must resort to a non-uniform grid near the walls.

To conclude this section we briefly discuss the initial conditions. Most simulations of laminar convection are started from the quiescent isothermal conditions expressed in (4) (and (8) in solving the non-Boussinesq equations). However, to expedite calculations, a different approach is used in transition studies. In this case, the initial conditions of an integration for a certain value of the Rayleigh number is obtained from the solution corresponding to a Rayleigh number which is close to it.

\section{Cavities with $A_{H} \geqslant 1$}

Although gas gaps between vertical parallel walls have been used for many decades to reduce heat transfer, their use with large horizontal temperature differences has become increasingly important during the last two decades. Examples of typical applications include insulation using double-pane windows or double walls, nuclear 
reactors, fire in buildings, solar collectors, and electronic components in enclosures. Still the majority of the published investigations involve small temperature differences where gas properties are taken to be constant and the Boussinesq limiting equations are valid. Here we briefly describe some of the known non-Boussinesq analytical, experimental, and numerical results in the laminar regime.

The first model of the flow in the cavity was proposed by Batchelor (1954) for small values of Rayleigh number. This model is not very valuable since these small values are well below values of practical significance. Gill (1966) subsequently proposed a boundary layer model in a two-dimensional cavity having large aspect ratios $\left(A_{H} \gg 1\right)$ and containing a fluid of large Prandtl number. Bejan (1979) completed Gill's boundary layer model by imposing adiabatic boundary conditions (and zero global heat flux) on the top and bottom boundaries, even though the boundary layer is not valid on these horizontal walls. The experimental studies of Eckert \& Carlson (1961) using air and Elder (1965) using silicone oil $\left(\operatorname{Pr} \approx 10^{3}\right)$ contributed a great deal to understanding the flow in cavities whose vertical aspect ratios varied between $2 \cdot 1$ and 46.7 and the Rayleigh numbers between $2 \times 10^{2}$ and $2 \times 10^{5}$. The work of Gill (1966) modified by Bejan (1979), was extended by Graebel (1981) who examined the influence of the Prandtl number. He demonstrated that the flow is only weakly influenced when the Prandtl number is of order unity.

Hara (1958), and Sparrow \& Gregg (1958) gave analytical results for a vertical flat plate with large temperature differences. In general their results are not applicable to the vertical slot; however, Chenoweth \& Paolucci (1986) have shown how their heat transfer results can be adapted to the differentially heated cavity. Polezhaev (1967) used equal Sutherland laws for dynamic viscosity $\mu$ and thermal conductivity $k$ (constant Prandtl number when the heat capacity at constant pressure is constant) in his numerical solution of the compressible Navier-Stokes equations; he used a relatively small temperature difference parameter of $\varepsilon=0.2$ and slot aspect ratios of unity. Rubel \& Landis (1970, pp. 1-11) made an expansion in terms of $\varepsilon$ to obtain first-order corrections to the zeroth-order Boussinesq results. They included fluid property variation by means of temperature power-law expressions and they assumed that the pressure was independent of the temperature difference as well as other parameters. Leonardi \& Reizes (1979, pp. 297--306, 1981, pp. 387-412) also numerically solved the compressible Navier-Stokes equations using equal Sutherland laws for $\mu$ and $k$; however they did examine cases where larger temperature differences were involved $(\varepsilon \leqslant 0.6$ ), and they studied only aspect ratios of 1 and 2 , although some of their results are suspect (see Chenoweth \& Paolucci 1986).

Experimental studies using gases with large temperature differences have been equally sparse. Eckert \& Carlson (1961) did investigate the high aspect ratio problem, but they only examined cases where $\varepsilon \leqslant 0 \cdot 13$, so that the Boussinesq solution is approximately valid for their results. Similarly, all of the Mordchelles-Regnier \& Kaplan (1963, pp. 94-111) experiments in the laminar region were in the Boussinesq regime, although they did obtain some turbulent non-Boussinesq results for a single flat plate. More recently, Duxbury (1979) obtained experimental results covering a wide parameter range in the steady laminar regime, but with $\varepsilon \leqslant 0 \cdot 15$. It is not clear to what extent the above experimental studies were affected by heat losses from the ends.

Chenoweth \& Paolucci (1985) investigated the steady-state fully developed boundary-layer region for large temperature differences between vertical isothermal walls. They derived some exact laminar solutions to the Navier-Stokes equations 
for perfect gases with the properties described by unequal Sutherland laws. They produced variable Prandtl number results for $0<\varepsilon<1$ using air as an example, although the accuracy of the Sutherland law conductivity degrades rapidly above $\varepsilon=0.6$.

Varying amounts of theoretical and experimental information relating to flow region classification in the Boussinesq limit and near Boussinesq limit can be found in Batchelor (1954), Bergholz (1978), Duxbury (1979), Eckert \& Carlson (1961), Lauriat (1980), Lee \& Korpela (1983), Mordchelles-Regnier \& Kaplan (1963, pp. 94-111), Polezhaev (1968), and Yin et al (1978). Much of the difference in the flow region bounds often can be related to the use of different criteria for the classification, although in some cases poor numerical resolution or experimental difficulties are responsible for the differences. A somewhat different picture will emerge if velocity field rather than thermal field or heat transfer information is used. Chenoweth $\&$ Paolucci (1986) present results for air using the ideal gas law and Sutherland law transport properties. Numerical solutions of the transient Navier-Stokes equations are used to generate laminar steady-state results primarily in the independent boundary layer region and the developing merged boundary layer region. However, other flow regions are also covered to the extent necessary to construct a better understanding of the entire laminar parameter range for all aspect ratios greater than or equal to unity. The velocity field behaviour is used to classify the different flow regions. The need to construct a map of flow regions in parameter space, which includes stationary and oscillatory stability boundaries, necessitated the use of the transient form of the equations. Wide ranges of aspect ratio, Rayleigh number, and temperature-difference parameters are examined. The results are compared in detail to the exact solution in the conduction and fully developed merged boundary layer limits for arbitrary temperature difference, and to the well-established Boussinesq limit for small temperature difference.

Figure 2 presents the flow region maps for $\operatorname{Pr}=0.71$ obtained from their extensive computations. The solid lines for the Boussinesq limit $\varepsilon \ll 1$ and the dashed lines for $\varepsilon=0.6$, which bound some of the regions, denote lines of stability. The limits of the boundary layer regimes are given by the dot-dash and the dotted lines, for $\varepsilon \ll 1$ and $\varepsilon=0.6$, respectively. The shaded region represents the unsteady transition to

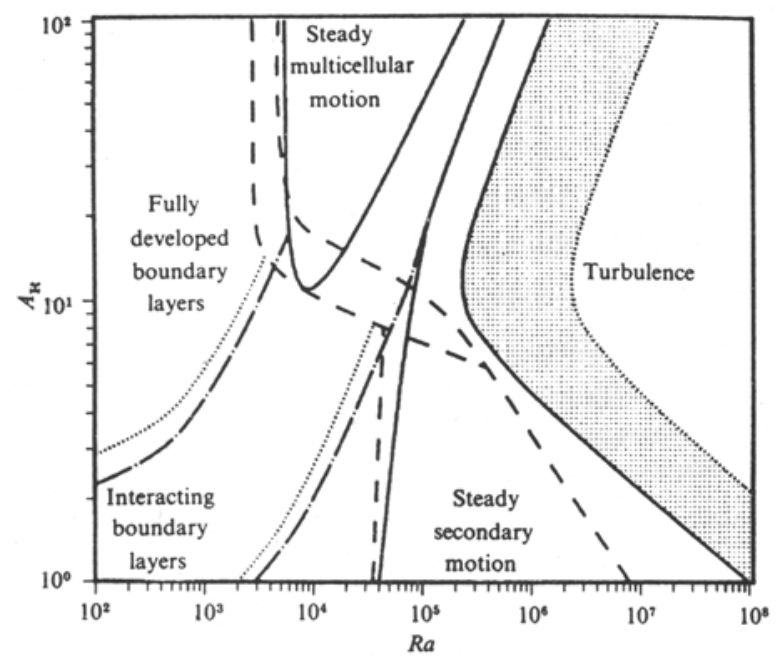

Figure 2. Flow regions dependence on $A_{H}, \mathrm{Ra}$ and $\varepsilon$ for $\operatorname{Pr}=0 \cdot 71$. 
turbulence for $\varepsilon \ll 1$. Correlations defining boundaries of the regions shown in figure 2 are given by Chenoweth $\&$ Paolucci (1986) and are discussed below.

The most familiar flow region, often called the boundary-layer region, is characterized by independent (non-interacting) boundary layers on the hot and cold walls; here the boundary layers are separated by a nearly stagnant core which always displays an approximately linear vertical thermal stratification. The boundary layer region exists primarily (although not exclusively) for low aspect ratios,

$$
1 \leqslant A_{H} \leqslant 12(1+4 \varepsilon) / \operatorname{Ra}^{0 \cdot 25 \varepsilon},
$$

and for Rayleigh numbers in the range

$$
7 \times 10^{3}\left(A_{H}-0.6\right)<\mathrm{Ra}<10^{4} A_{H}\left(1.5+\frac{10^{4}}{A_{H}^{4}}\right),
$$

for $\varepsilon \ll 1$, and

$$
4.5 \times 10^{3}\left(A_{H}-0.6\right)<\mathrm{Ra}<\frac{8 \times 10^{6} A_{H}^{-1.7}+5 \times 10^{3}\left(A_{H} / 12\right)^{6}}{1+\left(A_{H} / 12\right)^{6}}
$$

for $\varepsilon=0.6$. The upper limits in (18) and (19) represent critical Rayleigh numbers, since above those limits the flow no longer reaches a steady-state. When $\mathrm{Ra}$ is increased significantly above these critical values, the flow becomes increasingly unsteady and eventually becomes turbulent.

Below the lower limit of the independent boundary-layer region and for

$$
400\left[A_{H}\left(1-\varepsilon^{3}\right)-2\right]<\mathrm{Ra}<7 \times 10^{3}\left(1-\varepsilon^{2}\right)\left(A_{H}-0 \cdot 6\right),
$$

and aspect ratios approximately in the range (17), the velocity boundary layers are interacting to an increasing degree as the value of $\mathrm{Ra}$ is decreased. Finally for

$$
\mathrm{Ra}<400\left[A_{H}\left(1-\varepsilon^{3}\right)-2\right]
$$

fully developed merged boundary layers exist near the mid-height of the slot. At that point both velocity and temperature are within $3 \%$ of universal profiles which are independent of the vertical coordinate. As the aspect ratio is increased further, the fully developed profiles exist in a region whose size is proportional to $A_{H}$, since the development regions near each end remain nearly fixed in size for given $\mathrm{Ra}$ and $\varepsilon$. The fully developed region is centred about $A_{H} / 2$ for small $\mathrm{Ra}$, but for non-Boussinesq cases, when $\mathrm{Ra}$ is near the critical value of $5760(1+0.434 \varepsilon)^{-3}$ and $A_{H}>11$, it may be centred significantly below $A_{H} / 2$, since near that limit the bottom development region is approximately half that found at the top of the slot. For aspect ratios greater than 12-15, if the critical Rayleigh number of $5760(1+0.434 \varepsilon)^{-3}$ is exceeded, another type of instability can appear. This instability is characterized by standing waves and gives rise to multicellular flow inside the primary roll. Then combinations of large and small cells cover the entire slot and increase in strength with increasing $R a$. The number and size of these cells depend on $A_{H}, \mathrm{Ra}$ and $\varepsilon$. We note that in the Boussinesq limit these cells are approximately the same size, their number depends only on the aspect ratio, and their strength increases with increasing Ra (Lee \& Korpela 1983). This multicellular motion is completely steady.

In the Boussinesq limit, as the Rayleigh number is further increased, a region of 
reverse transition from multicellular to unicellular flow exists. This region is bounded by the multicellular region described by

$$
A_{H}>4.2 \times 10^{-3} \mathrm{Ra}^{1 \cdot 31}(\mathrm{Ra}-5760)^{-1 / 2},
$$

and by a thin boundary layer region containing steady secondary motion described by

$$
4 \times 10^{4} A_{H}^{1 / 4}\left(1+10^{-2} A_{H}^{5 / 4}\right)<\mathrm{Ra}<10^{4} A_{H}\left(1 \cdot 5+\left(10^{4} / A_{H}^{4}\right)\right) .
$$

Above the upper limit of (23), unsteady motion exists. The region of secondary motion described by (23) is in fact also valid for relatively low aspect ratios as shown in figure 2 and occupies most of the boundary layer region defined by (18). This is also true for $\varepsilon=0.6$ since for $A_{H}<8$ the lower limit of (23) is then replaced by

$$
\mathrm{Ra}>3.5 \times 10^{4} A_{H}^{1 / 8} .
$$

The reverse transition region, whose boundaries merge as $A_{H} \rightarrow \infty$ for $\varepsilon \ll 1$, does not exist for $\varepsilon=0.6$ since in this case the boundaries merge near the upper limit of (17) as shown in figure 2. In fact, in this case, the approximate expression (22) and the lower limit of (23) are now replaced by

$$
\mathrm{Ra}>2880+\left(40 / A_{H}\right)^{6 \cdot 67},
$$

so that strong multicellular motion is now present in this entire region. This large change for $A_{H} \gg 1$ and for $\varepsilon=0.6$ is accompanied by a greatly reduced critical Rayleigh number for unsteady motion as indicated by the upper limit of (19). The steady multicellular region is now confined to a narrow region between (25) and the upper limit of (19).

The location of the unsteady transition, displayed in figure 2 , is defined by the upper limit of (18). The results of Mordchelles-Regnier \& Kaplan (1963, pp. 94-111) for $A_{H}<12$ and extending down to aspect ratios as low as 1.67 , predict that the critical Rayleigh number varies with $A_{H}^{-1 / 2}$. Most of the disagreement with the results of Chenoweth \& Paolucci (1986) is at the lower aspect ratios where they show an $A_{H}^{-3}$ dependence. We note that as the aspect ratio is decreased, heat losses from the ends become more and more important and cause instabilities to occur at a lower value of Rayleigh number. Apparently, these end losses are responsible for the Mordchelles-Regnier \& Kaplan (1963, pp. 94-111) weaker dependence on aspect ratio. Quantitative agreement is much better with results obtained by linear stability analysis by Bergholz (1978). In making this comparison, use is made of the fact that the temperature in the core is approximately linearly stratified with a stratification parameter which is near unity in this region. Bergholz's results do show that the critical Rayleigh number varies approximately with aspect ratio as $A_{H}^{-3}$ for $A_{H}<6$. The result obtained from Bergholz's analysis arises from a travelling wave instability along the side walls. However, Chenoweth \& Paolucci (1986) offer another possible explanation for the $A_{H}^{-3}$ behaviour due to a "hydraulic" jump instability on the end walls. The experimental results of Ivey (1984) for $\operatorname{Pr}=7 \cdot 1$ and $A_{H}=1$ clearly indicate the presence of a "hydraulic" jump and a stationary wavetrain downstream of it when the Rayleigh number is above the critical internal Froude number. His observations are in complete agreement with those of Paolucci \& Chenoweth (1989).

Bergholz's (1978) results also show that there is a minimum critical Rayleigh number for oscillatory instability, occurring at $1.15 \times 10^{5}$ at an aspect ratio near 12 . 
This compares well with the minimum of $\mathrm{Ra}=2.4 \times 10^{5}$ also occurring near $A_{H}=12$ shown in figure 2. At aspect ratios larger than 12 the results of Chenoweth \& Paolucci (1986) show that the stratification parameter is nearer 0.5, and the critical Rayleigh number is approximately linear with $A_{H}$. This picture is in good agreement with the travelling wave instability from steady to unsteady motion given by Bergholz (1978), and consistent with experimental data of Mordchelles-Regnier \& Kaplan (1963, pp. 94-111) for $12 \leqslant A_{H} \leqslant 25$.

Results of Lee \& Korpela (1983), and Lauriat (1980) show that in the Boussinesq limit, for $\mathrm{Ra} \approx 10^{4}$ the flow changes from unicellular to multicellular motion as the aspect ratio is increased. They observe the transition occurring near $A_{H}=11-12$, in agreement with the minimum aspect ratio shown in figure 2.

Still in the Boussinesq regime, Bergholz (1978) also obtained the critical Rayleigh number corresponding to the standing wave or stationary transition from fully developed merged boundary layers to steady multicellular motion. He obtained this value as approximately 5740 , which again compares very well with the results,

for

$$
\mathrm{Ra}<\mathrm{Ra}_{c}=5760(1+0 \cdot 434 \varepsilon)^{-3},
$$

$$
A_{H}>A_{H_{d}}=(2+\mathrm{Ra} / 400) /\left(1-\varepsilon^{3}\right),
$$

given by Chenoweth \& Paolucci (1986) in the limit $\varepsilon \rightarrow 0$. However, Lee \& Korpela (1983), using 17 grid points in the horizontal direction, obtain this transition between 7100 and 7810 for $A_{H}=20$, which is much higher than the result shown in the figure and Bergholz's result. They explain this disagreement by noting that for large but finite aspect ratios of order 20 , there is a small positive vertical stratification through the cavity, since the boundary layers are not yet completely developed, and Bergholz shows that the flow is stabilized with increasing stratification. Even though this argument correctly explains why the critical Rayleigh should increase for lower aspect ratios, figure 2 does not show a substantial increase until $A_{H}<20$. In fact, the calculations of Chenoweth \& Paolucci (1986) for $\mathrm{Ra}=7100$ and $A_{H}=20$ show multicellular motion, in agreement with the results of Roux et al (1980) obtained by using a higher order scheme. A better explanation for the disagreement is that Lee $\&$ Korpela (1983) lacked sufficient horizontal resolution. The lower bound on the fully developed boundary layer region $A_{H}>\mathrm{Ra} / 500$ given by Batchelor (1954) agrees with figure 2 for large $\mathrm{Ra}$ but differs substantially at low $\mathrm{Ra}$ since fully developed boundary layers at the mid-height plane cannot be developed for $A_{H}<2$ even if $\mathrm{Ra}$ and $\varepsilon$ approach zero.

Polezhaev (1968) constructed a somewhat complete picture of the flow regions for the same $A_{H}$ and Ra ranges as shown on figure 2 . In spite of the very coarse grids used, the regions where calculations were made and those where he used data from Eckert \& Carlson (1961) as well as Elder (1965a) are in qualitative agreement with figure 2. However, in the regions where he extrapolated the bounds, there is substantial disagreement, particularly where both $A_{H}$ and $\mathrm{Ra}$ are large and where both are small. The same statement applies to the regions plot given by Yin et al (1978), since only a band of results extending from low $\mathrm{Ra}$ and high $A_{H}$ to high $\mathrm{Ra}$ and low $A_{H}$ were used to locate lines which were then extrapolated across the other regions.

Roux et al (1980) found, in the Boussinesq limit, that a small region of reverse transition from multicellular to unicellular flow exists within the lower right corner of the region defined by $A_{H} \geqslant 12$ and $10^{4} \leqslant \mathrm{Ra} \leqslant 10^{5}$. We note that this region 
corresponds to the narrow region separating stationary and oscillatory stability branches, for a narrow range of stratification parameter, as given in figure 3 of Bergholz (1978). Chenoweth \& Paolucci (1986) have verified the existence of this narrow region for $\varepsilon \ll 1$. However, as $\varepsilon$ increases, this region quickly disappears, and as suggested by the dashed line in figure 2 , it does not exist for $\varepsilon=0.6$.

The influence of the Prandtl number is found to be minor when $\operatorname{Pr}>1$. For $\operatorname{Pr}<1$, however, the velocity field and heat transfer rates have a strong dependence on $\mathrm{Pr}$. Quon (1972) performed finite difference calculations for the boundary layer regime in a square cavity in the Boussinesq limit. Consistent with the results of MacGregor \& Emery (1969) he showed that the flow in this regime is insensitive to the magnitude of the Prandtl number for $7 \cdot 14<\operatorname{Pr}<900$. Korpela et al (1973) report that when the Rayleigh number reaches the critical value, the conduction regime may become unstable in two ways. For $\operatorname{Pr}<12 \cdot 7$, the instability sets in as vertical stationary cells, with the critical Rayleigh number nearly independent of Pr. When $\operatorname{Pr}>12 \cdot 7$, the instability is manifested in the form of travelling waves. More recently, Lee \& Korpela (1983) carried out extensive numerical computations of multicellular flows in cavities of aspect ratios as large as 40 for a wide range of Prandtl numbers in the Boussinesq limit. They found that for low Prandtl number fluids $(\operatorname{Pr} \ll 1)$ such as liquid metals, multicellular flows can develop when the aspect ratio is as low as 6 . For air $(\operatorname{Pr}=0.71)$, the aspect ratio must be at least 12 before the flow becomes more complex as a result of instability. These results are consistent with the stability results of Bergholz (1978).

Very few numerical calculations have been performed in three-dimensional cavities. Of these we note the works of Mallinson \& de Vahl Davis (1977) and Lee et al (1988) with cavities having $A_{H}=1$ and $A_{D}$ varying from 2 to 4 , and the most recent results of Fusegi et al (1991) with $A_{H}=A_{D}=1$. Because of the large computational costs, all such calculations have been limited to $\mathrm{Ra} \leqslant 10^{6}$. The exception is the work of Lankhorst \& Hoogendoorn (1988) who computed the flow in enclosures with $A_{H}=1$ and $A_{D}=1$ and 2 for Rayleigh numbers as large as $10^{10}$. However, we note that their finite difference meshes were very coarse $(45 \times 45 \times 20)$ and they made use of a $k-\varepsilon$ turbulence model. As will be seen later, it appears highly unlikely that such models, which were developed for high Reynolds number flows, are applicable to low Reynolds number natural convection turbulence. Comparisons obtained from resolved $\left(\mathrm{Ra} \leqslant 10^{6}\right)$ laminar results at the symmetry, plane $(z=0)$ show that peak minimum and maximum velocities and heat transfer rates are within $10 \%$ of values obtained from two-dimensional calculations. Average values of the Nusselt number actually agree within $2 \%$. In general the agreement is better at the larger values of Rayleigh numbers. Thus the major conclusion is that at high Rayleigh numbers, threedimensional effects are insignificant in the bulk of the flow field. The exceptions are the regions near the end walls.

\section{Cavities with $A_{H} \leqslant 1$}

Cavities with small aspect ratios have not received as much attention as those with aspect ratios larger than unity. Furthermore, most analytical and experimental studies conducted to date on this problem have been in the Boussinesq or near-Boussinesq limit.

In exemplary studies, the problem was treated by Cormack et al (1974a, 1.974b) and Imberger (1974) analytically, numerically, and experimentally, respectively. 
Cormack et al (1974a) presented an asymptotic solution to the laminar problem. This solution is valid in the aspect ratio limit of $A_{H} \rightarrow 0$ for fixed, though arbitrary values of the Rayleigh and Prandtl numbers, and is assumed to consist of two distinct regions: a parallel flow in the central core (first obtained by Hart 1972) and a non-parallel flow confined within a distance of order $H$ from the end walls. Cormack et al (1974b) developed an algorithm for the numerical solution of the flow in the cavity. They found their results to be in good agreement with the parallel flow solution obtained by Cormack et al (1974a) provided $A_{H} \leqslant 0 \cdot 1$ and $\mathrm{Ra}^{2} A_{H}^{3} \leqslant 10^{5}$. In addition, the solutions show a parallel flow transition between the asymptotic limit of Cormack et al (1974a) and the boundary layer limit $\left(A_{H}\right.$ fixed and $\left.\mathrm{Ra} \rightarrow \infty\right)$ of Gill (1966). Imberger (1974) presented experimental results for water with $A_{H}=0.01$ and 0.019 in the range $1.31 \times 10^{6} \leqslant \mathrm{Ra} \leqslant 1.11 \times 10^{8}$. Most of the flow features indicated by the numerical work were qualitatively observed in the experimental work. When $\mathrm{Ra}^{2} A_{H}^{3}$ becomes nearly $10^{11}$, experiments indicate that the mid-height of the cavity becomes an isotherm and there is a slow central circulation throughout the entire shallow cavity. We note that while $A_{H}$ can be decreased by increasing the length of the cavity without varying the Rayleigh number, often to change the aspect ratio in an experiment the height is decreased thus forcing a large increase in temperature difference to keep the Rayleigh number fixed. Use of this experimental procedure to verify numerical Boussinesq results has limited validity since the numerical solutions do not account for property variations.

Bejan \& Tien (1978) and Bejan (1980) extended the asymptotic analysis of Cormack et al (1974a) to include both $\mathrm{Ra} \rightarrow 0, A_{H}$ finite, and $A_{H} \rightarrow 0$, Ra finite. They also developed a Nusselt number correlation in the boundary layer regime and an empirical correlation including both limits. Bejan et al (1981) presented experimental results for water with $A_{H}=0.0625$ and $2 \times 10^{8}<\mathrm{Ra}<2 \times 10^{9}$. They showed that, contrary to the assumption of Bejan \& Tien (1978), for $\mathrm{Ra}^{1 / 4} A_{H}>1$, the core flow is non-parallel and is dominated by horizontal intrusions flowing along the two insulated horizontal walls and embracing a practically stagnant and thermally stratified fluid. In addition they observed weak counterflow. Finally, by statistically analysing previously published experimental work, Ostrach (1982), in a recent review paper, shows that the heat transfer varies with $\mathrm{Ra}^{0.3}$ for $A_{H}<0.1$ and $\mathrm{Ra}>5 \times 10^{5}$. He notes that the variation with $\mathrm{Ra}^{0 \cdot 2}$ obtained by Bejan \& Tien (1978) is incorrect since they assumed that the core flow was parallel in matching with the boundary layers in the end regions.

Paolucci \& Chenoweth (1988) improved the understanding of the small aspect ratio problem. They emphasize non-Boussinesq effects arising from property variations due to large temperature differences. In addition, they extend the numerical solution in the Boussinesq regime to Rayleigh numbers larger than those previously available. Numerical solutions of the transient Navier-Stokes equations are used to generate laminar steady-state results. The use of the transient form of the equations was necessary to obtain oscillatory stability results. Their results are summarized in figure 3 where the various flow regions are classified according to different velocity field behaviours. This figure is the result of many calculations and is given in $A_{H}-\mathrm{Ra}$ space for $\operatorname{Pr}=0.71$ and two values of $\varepsilon$; the solid lines are for $\varepsilon \rightarrow 0$ and the dashed line is for $\varepsilon=0 \cdot 6$. The numbered solid lines bound the different flow regions.

To the left of I the core flow is parallel. Cormack et al (1974a), and Hart (1972) give the exact solution for this flow. It can be easily shown that in this region the maximum horizontal velocity is $u_{m}= \pm \mathrm{Ra} / 72 \sqrt{3}$ and its location is at $y_{m}=(1 \mp 1 / \sqrt{3}) / 2$. In the region between I and II, parallel flow still exists in the core, but the magnitude 


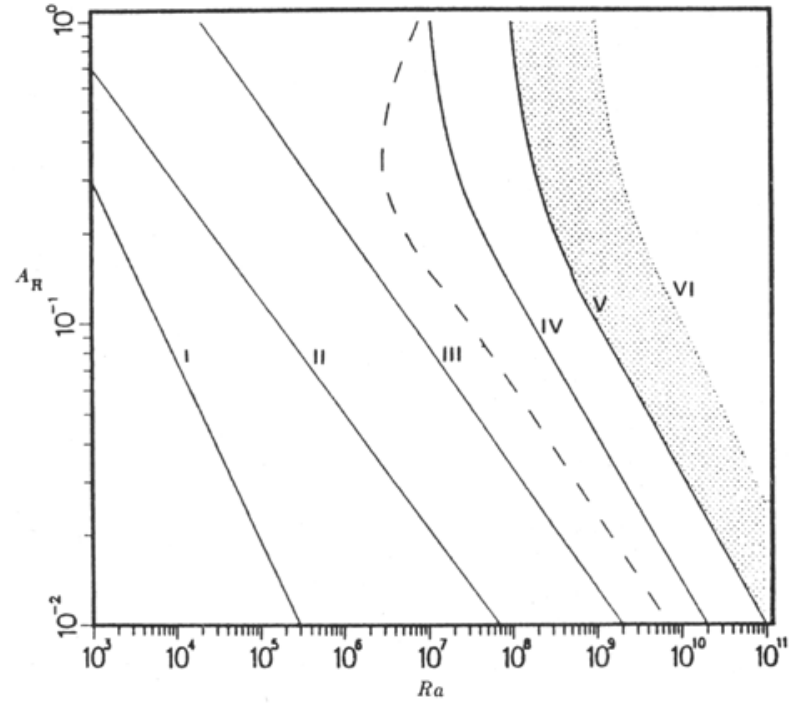

Figure 3. Flow regions dependence on $A_{H}, \mathrm{Ra}$ and $\varepsilon$ for $\operatorname{Pr}=0.71$.

of the maximum velocity decreases below $u_{m}$, even though its location remains the same. Cormack et al (1974a) as well as Bejan \& Tien (1978) give formulas for the variation of $u_{m}$ which depend on aspect ratio and Rayleigh number. The flow remains unicellular between II and III, but the core flow becomes non-parallel and boundary layers exist next to the active walls. Thus all regions to the right of II are considered to be in the boundary layer regime. In the region between III and IV, secondary flows develop in the top-left and bottom-right (emerging) corner regions. These secondary flows separate and elongate as the Rayleigh number is increased and eventually grow to occupy most of the core region. The flow remains steady in the region between IV and V, but a weak tertiary flow develops in the centre of the core region. The rotation of this cell is the same as those of the primary and secondary flows. Finally to the right of $\mathrm{V}$ the flow becomes unsteady and eventually becomes turbulent to the right of VI. The unsteady flow is in the form of oscillations near the active walls. These oscillations, and resulting turbulence, are localized near the endwalls and emerging corners, and do not penetrate the central core region. We remark that throughout the entire boundary layer regime, the temperature in the central core region is approximately linearly stratified with horizontal isolines.

For $\varepsilon=0.6$ the flow is unicellular approximately up to line III. However, in contrast to the Boussinesq limit, no parallel flow was observed for this case which has the character of either that to the left or right of I. Due to property variations, the flow on the cold wall enters the boundary layer regime long before II, while that at the hot wall makes the transition significantly to the right of II. Similarly, secondary flows develop near the emerging corners at the cold end significantly before III and at the hot end much later than III. A most important result is that at the dashed line the cold wall boundary layer becomes unsteady. This same transition corresponds to line $\mathrm{V}$ in the Boussinesq limit. Note that there is very large reduction in the critical Rayleigh number with increasing $\varepsilon$. In contrast, the hot wall layer remains steady well past line V. Similarly, there is a large $\mathrm{Ra}$ range where the flow at the cold wall end becomes turbulent, but the hot wall region and the core flow remain laminar since the turbulence is localized and does not penetrate very far into the central core. 
Contrary to the results of Shiralkar et al (1981), and Tichy \& Gadgil (1982), Paolucci $\&$ Chenoweth (1988) find the presence of weak reverse flows in the core region for high $\mathrm{Ra}$ in the boundary layer regime, in agreement with the experimental results of Bejan et al (1981) and Al-Homoud \& Bejan (1979). In addition, for still higher Ra, Paolucci \& Chenoweth (1988) observe this flow bifurcating and leading to a tertiary flow consisting of a central cell having the same rotation as the primary and secondary cells. This last result has not been previously observed either experimentally or numerically.

In the non-Boussinesq regime, the velocity and temperature fields results of Paolucci \& Chenoweth (1988) show significant dependence on $\varepsilon$ especially near the side walls. As a result the well-known parallel flow solution, accurate in the core of the cavity for $\varepsilon \ll 1$, does not exist. For higher Rayleigh numbers, Paolucci \& Chenoweth (1988) generalize the well-known analytical boundary layer solution of Gill (1966) to the case of arbitrary $\varepsilon$. Their solution and numerical results show that the cold-wall boundary layer is much thinner than the corresponding layer in the Boussinesq limit. As a result, the critical Rayleigh numbers for stationary and oscillatory instabilities are lowered with increasing temperature difference and are governed by the cold wall. In contrast to the high aspect ratio problem (see Chenoweth \& Paolucci 1986), they find that the heat transfer and pressure also depend strongly on $\varepsilon$. Although the average Nusselt number is almost independent of $\varepsilon$ in the boundary layer regime, this is not the case for lower Rayleigh numbers. Furthermore, the largest pressure change occurs in this same low Rayleigh number region. Both effects are physically related to the large departures from the parallel flow solution valid in the Boussinesq limit. These results show that there can be considerable risk if results obtained from the well-established Boussinesq limit are extrapolated for use where large temperature differences exist.

As in the case where $A_{H}>1$, when the Prandtl number is of order unity or larger, the above results change little (see Quon 1972). However, when the Prandtl number is small, substantial changes appear. Hart (1983) and more recently Drummond \& Korpela (1987) show that for $\operatorname{Pr}<0.12$, and aspect ratios less than the same value, a parallel flow core will exist up to $G r \approx 8000$ where secondary flow in the form of stationary transverse cells is seen to appear. These cells propagate out from the ends as an imperfect bifurcation. Spacing of the cells in the cavity has been shown to depend on $\mathrm{Gr}, \operatorname{Pr}$ and $A_{H}$. Cases were found for which new cells form and grow between existing cells as the space permitted. Other cases show cells merging and splitting. The effect of increasing $\operatorname{Pr}$ is to stabilize the flow so that for $\operatorname{Pr}>0.12$ with $A_{H}=1 / 8$ and $1 / 10$ no secondary motions were found.

\section{Transition of boundary layer flow}

The majority of the work dealing with differentially heated cavities has been concerned with steady-state laminar flow. Yet in many of the fields of application, the flow is unsteady and possibly turbulent. Since many variables of engineering interest depend strongly on the flow regime, it is essential to understand the different physical processes responsible for the conversion of an initially laminar flow to a turbulent one. With increasing Rayleigh number the problem becomes stiff owing to a decrease in boundary layer thickness. As a result, there has been very little numerical work performed in this area. 
The study of stability of the natural convection flow in the differentially heated cavity presents a major difficulty: the non-existence of an exact analytical solution in the general case. Studies have proceeded by extrapolating results obtained in studying limiting cases where solutions can be obtained. These are cases that we have encountered earlier and are known as the conduction limit, the buoyancy layer, and the separate boundary layer regime. The latter case is characterized by the presence of a stable vertical temperature stratification. Such stability studies have been conducted by Gill \& Davey (1969) for the buoyancy layer and Vest \& Arpaci (1969) and Bergholz (1978) for separated boundary layers, on assuming two-dimensional perturbations. In these studies, the fluid is assumed to be linearly stratified. It was found that below a certain stratification level the instability was stationary, while above this critical stratification level it was oscillatory. Korpela (1974) studied the influence of the Prandtl number on the stability of the conduction regime. We note here that in order to relate these results to the closed cavity, the stratification level needs to be empirically related to $A_{H}$ and $\mathrm{Ra}$. Furthermore, the stratification is applied uniformly in the width of the cavity up to and including the active walls. Obviously this represents an approximation to the actual situation, and the extent of applicability to the differentially heated cavity can only be judged by comparison to full numerical studies of stability. In addition, Paolucci \& Chenoweth (1989) show that their results applied to finite cavities (using a thermal stratification parameter near unity) yield a critical wavelength of the vertical wall boundary layer $\lambda_{c} \sim A_{H} / \operatorname{Pr}^{n}$, where $n \leqslant 1 / 2$; thus the applicability of their results to finite cavities is questionable for small Prandtl numbers since Paolucci \& Chenoweth (1989) find $\lambda_{c} \approx 0.3 A_{H}$ for $\operatorname{Pr}=0.71$, where $n \approx 0$. Lastly, these analyses cannot yield any possible instabilities due to the presence of the horizontal walls. Iyer (1973) showed that two-dimensional transverse waves are the most unstable in the buoyancy layer, thus indicating that a two-dimensional assumption is not unreasonable. However, it is not clear that this assumption will remain valid in the nonlinear regime. Patterson \& Imberger (1980) were the first to propose a classification of types of transition regimes that one could encounter within the cavity. Depending on whether the Prandtl number is larger or smaller than unity, the authors discuss the time to establish steady solutions, when they exist, and the diverse states in which the flows evolve to the stationary solutions. They also give conditions for the existence of the separate boundary layer regime. Patterson \& Imberger (1980) and Patterson (1984) gave a criterion for the presence of internal gravity waves that are observed in the core of the cavity when $\operatorname{Pr} \geqslant 1$ and $A_{H} \leqslant 1$. The presence of these gravity waves in the cavity is also discussed by Yewell et al (1982), Ivey (1984), and Thorpe (1968) who studied stationary gravity waves in fluids in the presence of continuous and discontinuous stratifications. Patterson \& Imberger (1980) concluded that cavity-scale internal wave activity is due to a "pile up" of the horizontal intrusions at the far ends. Ivey (1984) performed experiments in a square cavity at Rayleigh numbers of the order of $10^{9}$ using water as the working fluid. He emphasized the importance of the inertial effect of the flow and his results show that damped oscillations arise from internal hydraulic jumps caused by the turning of the vertical boundary layers. Since the source is localized, he further concludes that due to rapid attenuation, their presence could not be felt throughout the cavity as Patterson \& Imberger (1980) suggest. The numerical results of Chenoweth \& Paolucci (1986) seem to be in agreement with Ivey's (1984) conclusions; furthermore, they also suggest that for low aspect ratios the "hydraulic" jumps are responsible for the first transition to time-dependent flow. 
Le Quéré \& Alziary de Roquefort (1985) used a semi-implicit Chebyshev pseudospectral method to examine the oscillatory approach to steady-state of the average Nusselt number for $A=1, \operatorname{Pr}=0.71$, and $\mathrm{Ra}=10^{7}$ and $4 \times 10^{7}$. For $\mathrm{Ra}=4 \times 10^{7}$ they note the presence of detached regions near the departing corners at steady-state. These regions were also present at $\mathrm{Ra}=10^{7}$, but in this case they did not persist to steady-state. Subsequently, Le Quéré \& Alziary de Roquefort (1986, pp. 1532-37) computed the first transition to periodic flow for $A_{H}$ as low as 2 and concluded that in all cases the time-dependent periodic motion is a result of wall boundary layer instability. However, for $A_{H}<-3$ they did note the presence of separated flow regions along the horizontal walls which remained when the flow was observed to be statistically stationary. Haldenwang (1986), also using a semi-implicit Chebyshev pseudo-spectral method, computed the solution for $A_{H}=1, \operatorname{Pr}=0.71$, and $\operatorname{Ra}=10^{6}$, $10^{6.5}, 10^{7}, 10^{7.5}, 10^{8}$, and $10^{8.5}$. He concluded that: regions of reverse flow on the horizontal walls are present for $\mathrm{Ra} \geqslant 10^{7.5}$; the flow becomes oscillatory for $\mathrm{Ra}$ between $10^{8}$, and $10^{8 \cdot 5}$ where two fundamental frequencies were observed; and that these two frequencies, neither of which is in continuity with the one observed at smaller Rayleigh numbers, are first observed in the stable solution at $\operatorname{Ra}=10^{8}$.

Paolucci \& Chenoweth (1989) address the oscillatory approach to steady-state and the transitions from steady-state to turbulence via two-dimensional direct numerical simulations. Their results clarify the basic mechanism of steady and unsteady oscillatory motion. Simulations are performed for a Boussinesq fluid with $\operatorname{Pr}=0.71$, $1 / 2 \leqslant A_{H} \leqslant 3$, and a wide range of Rayleigh numbers. The resulting accuracy of their results is demonstrated by the excellent agreement with the results of Le Quéré \& Alziary de Roquefort (1986), and Haldenwang (1986). Their restriction to two spatial dimensions precludes possibly important three-dimensional nonlinear effects due to vortex stretching. But even within their limitations, imposed by present day computers, the simpler model is of interest in providing insight into the physical mechanisms which drive the convective dynamics from laminar to turbulent flows. Furthermore, while it is an accepted fact that the laminar flow is inherently two-dimensional (see Eckert \& Carlson 1961), some experiments indicate that even the resulting turbulent flow is dominated by two-dimensional structures (e.g. Elder 1965b). The goal behind their numerical experiments was to study the transitions to various time-dependent flows. With increasing Rayleigh number the onset of periodic flow is calculated for various aspect ratios. Power spectra of the temperature and velocity components are examined, and their dependence on the location probed is discussed. They are primarily concerned with instabilities that precede turbulence rather than strongly turbulent flows, although they also look at the oscillatory approach to steady-state for high Rayleigh numbers. The study of the loss of stability to time-dependent flow by direct numerical simulation allows them to obtain solutions for large supercritical values of the Rayleigh number. All their simulations satisfy a criterion for the presence of internal wave activity similar to that of Patterson \& Imberger (1980), but for $\operatorname{Pr} \leqslant 1$ and arbitrary $A_{H}$.

As a result of the numerous computations for various values of $A_{H}$ and $\mathrm{Ra}$, Paolucci $\&$ Chenoweth (1989) obtain the stability map displayed in figure 4 . This figure is a more detailed and accurate stability map of the lower right corner of figure 2 . The solid and dashed lines in the figure represent the critical Rayleigh numbers $\operatorname{Ra}_{i}$ due to internal waves and $\mathrm{Ra}_{w}$ due to the wall boundary layers respectively. To the left of the curves, perturbations are damped, while to the right they are amplified leading to oscillatory flow. With increasing Rayleigh number, the steady convection flow 


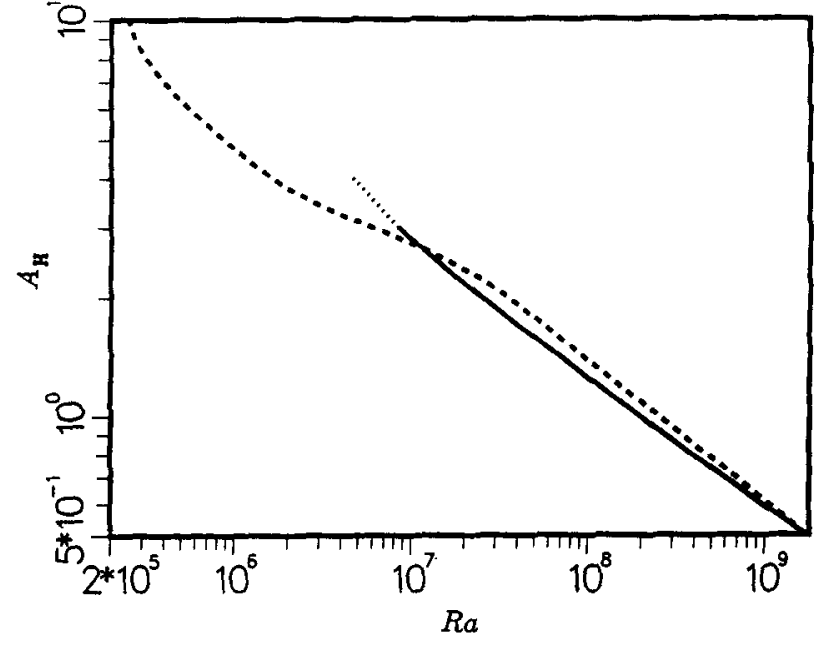

Figure 4. Critical Rayleigh numbers as functions of aspect ratio $A_{H}$ for $\varepsilon \rightarrow 0$ and $\operatorname{Pr}=0.71$ :

becomes unstable with respect to time-dependent disturbances. In general, for $A_{H} \leqslant 1 / 2$ and $A_{H} \geqslant 3$ the first time-dependent instability is due to the boundary layers along the vertical walls. However, for $1 / 2<A_{H}<3$ they first find periodic motion due to internal waves near the departing corners, then quasi-periodic motion (arising from wall boundary layers), aperiodic motion with complex regularity, and finally turbulent motion. They discuss at length the physical mechanisms responsible for the dynamic behaviours. Their results clearly show that for $\varepsilon \rightarrow 0, \operatorname{Pr}=0.71$ and $1 / 2<A_{H}<3$ internal waves near the departing corners are indeed the source of low frequency oscillations as suggested by Ivey (1984), and not caused by a "pile up" of the horizontal intrusions at the far ends as argued by Patterson \& Imberger (1980). While the high frequency oscillations are rapidly attenuated, the low frequency ones are not, so that their presence is felt throughout the cavity. The low frequency attenuation however is not in general as rapid as suggested by Ivey's (1984) experiment with water since their presence is felt throughout the cavity. In the range $1 / 2<A_{H}<2$ the curves in figure 4 are described to a good approximation by

and

$$
\mathrm{Ra}_{\mathrm{i}}=1.93 \times 10^{8} A_{H}^{-3.15} \text {, }
$$

$$
\mathrm{Ra}_{w}=2.70 \times 10^{8} A_{H}^{-2.75} \text {. }
$$

They also show that (28) and (29) are consistent with estimates obtained by the use of simple arguments and previous analyses.

In accord with Patterson \& Imberger (1980) they show that within this region of parameter space the flow approaches steady-state conditions in an oscillatory fashion, although the source of the oscillations is different from that suggested by them. In agreement with Ivey's (1984) experimental results they find that the oscillatory behaviour is due to the inertia of the flow entering the interior of the cavity from the sidewall boundary layers (departing corners), which leads to a form of internal "hydraulic" jump when the Rayleigh number is sufficiently large. The onset and frequencies of the oscillatory instabilities are calculated and compared with available data. They present numerical experiments which exhibit repeated supercritical 
branching leading to chaotic flow after a finite number of bifurcations. A sequence of instabilities quite similar to that described in their paper has been observed by Gollub \& Benson (1980) in a laser-Doppler velocimetry study of Rayleigh-Bénard convection and also by Fenstermacher et al (1979) and Gorman \& Swinney (1982) in the Couette-Taylor system. In particular, as seen in figure 5 , with increasing Rayleigh number the time history and spectra show a periodic regime with a single fundamental frequency followed by a quasiperiodic regime with two fundamental frequencies, and then broadband noise components appear in the spectra; finally the amplitude of the sharp frequency components decrease. This particular sequence of bifurcations into chaos is further illustrated by the phase-space trajectories shown in figure 6. Thus, one principal finding of this work is that periodic flow is followed by only one additional distinct dynamical regime (quasi-periodic flow with two incommensurate frequencies) prior to the appearance of a chaotic regime. This result is consistent with the predictions of Newhouse et al (1978). In addition, even though they restricted the simulations to two spatial dimensions, their results compare favorably with the experimental results of Ivey (1984), and analytical/experimental studies of Thorpe (1968), and Keunecke (1970).

Briggs \& Jones (1985) were the first to demonstrate experimentally different flows for the same values of parameters in a closed cavity having conducting horizontal walls. They demonstrated that in the Rayleigh number interval between $6 \times 10^{6}$ and $8 \times 10^{6}$ a hysteresis is present and a temporally oscillatory flow having one of three different frequencies can occur.

We remark that for $A_{H}=O(1)$ and small Prandtl numbers, it becomes experimentally difficult to maintain adiabatic boundary conditions on the horizontal walls as Ra becomes large. Furthermore, in a physical experiment, often the Rayleigh number is increased by increasing the temperature difference across the cavity. It has been shown that both of these effects greatly modify the stability of the flow (Chenoweth \& Paolucci 1986; Le Quéré \& Alziary de Roquefort 1986).

\section{Turbulence}

While there has been substantial work devoted to the study of turbulent natural convection in the Rayleigh-Bénard problem where the gravitational vector is parallel to an imposed thermal gradient, relatively minor attention has been given to the case where gravity is orthogonal to the gradient. There are only a few publications on this subject and primarily in the Boussinesq regime. Furthermore, the published experimental data are insufficient to fully characterize the mechanisms responsible for momentum and heat transport in the thermal layers. In contrast, theoretical and experimental studies for the same problem but in the laminar regime are abundant. For example, see Elder (1965a), Cormack et al (1974a), De Vahl Davis \& Jones (1983), Chenoweth \& Paolucci (1986), and references therein.

In a vertical layer that is bounded by vertical isothermal surfaces having different temperatures and is thermally insulated at the ends, a circulatory flow is set up, ascending against the hot surface and descending at the cold surface. The flow in the cavity passes through several stages as the fluid flows along the active vertical walls. The flow near the entry corners of the boundary layers is initially laminar. It then passes through a transition region, and finally becomes turbulent. When statistical steady-state obtains, the space between the vertical boundary layers is filled by a 

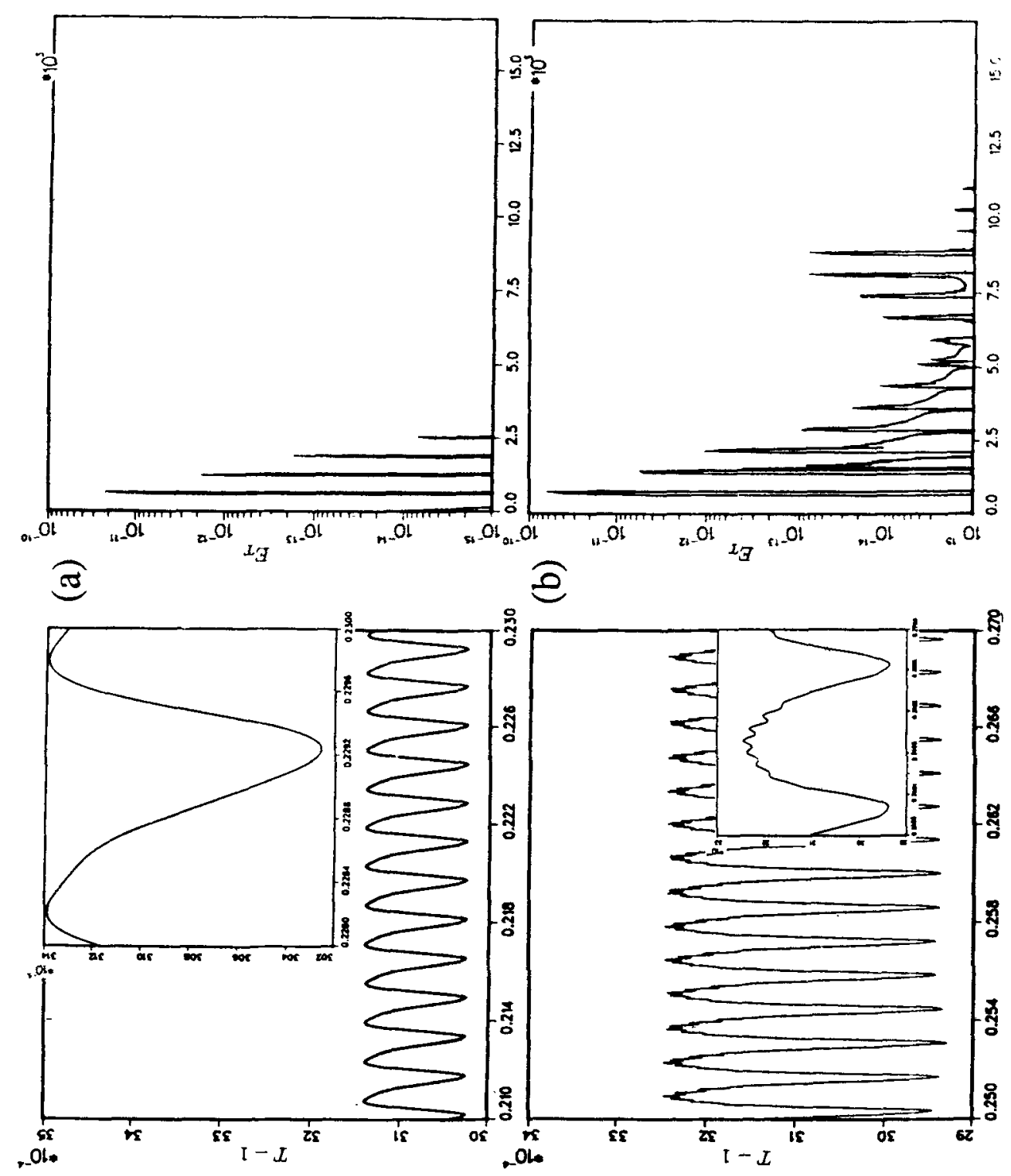


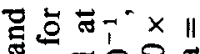
프으울 包宁 $\times 11$ o卜 类

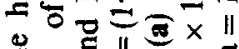
政宅

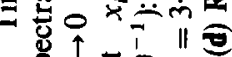
ن

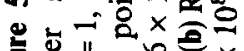

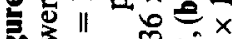
蛋

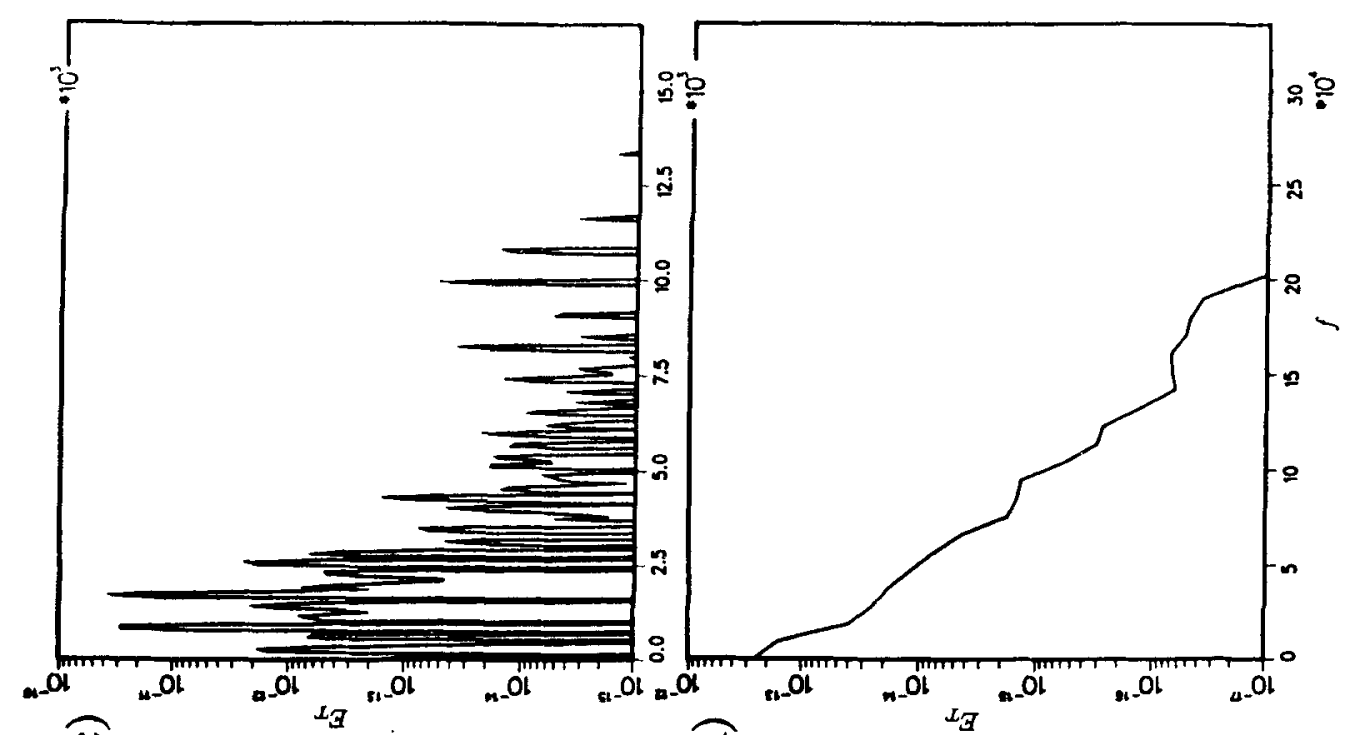
U ?
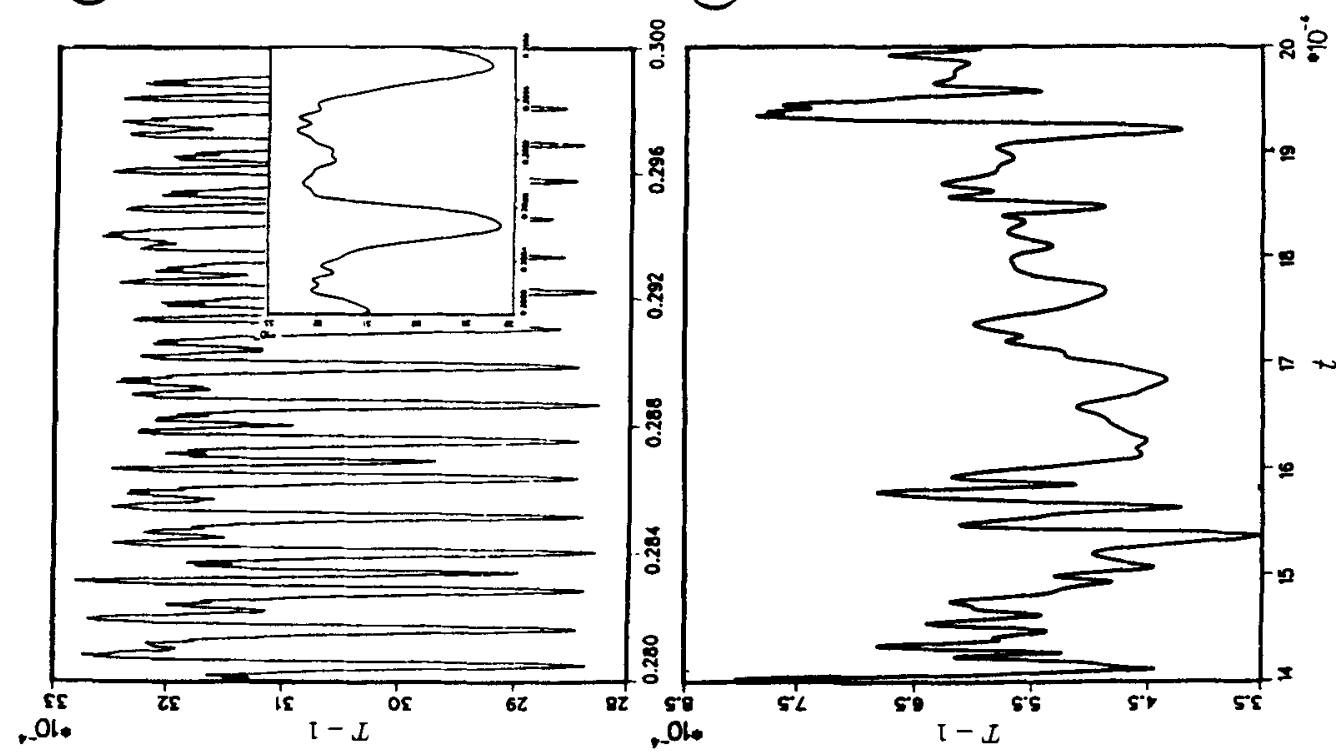

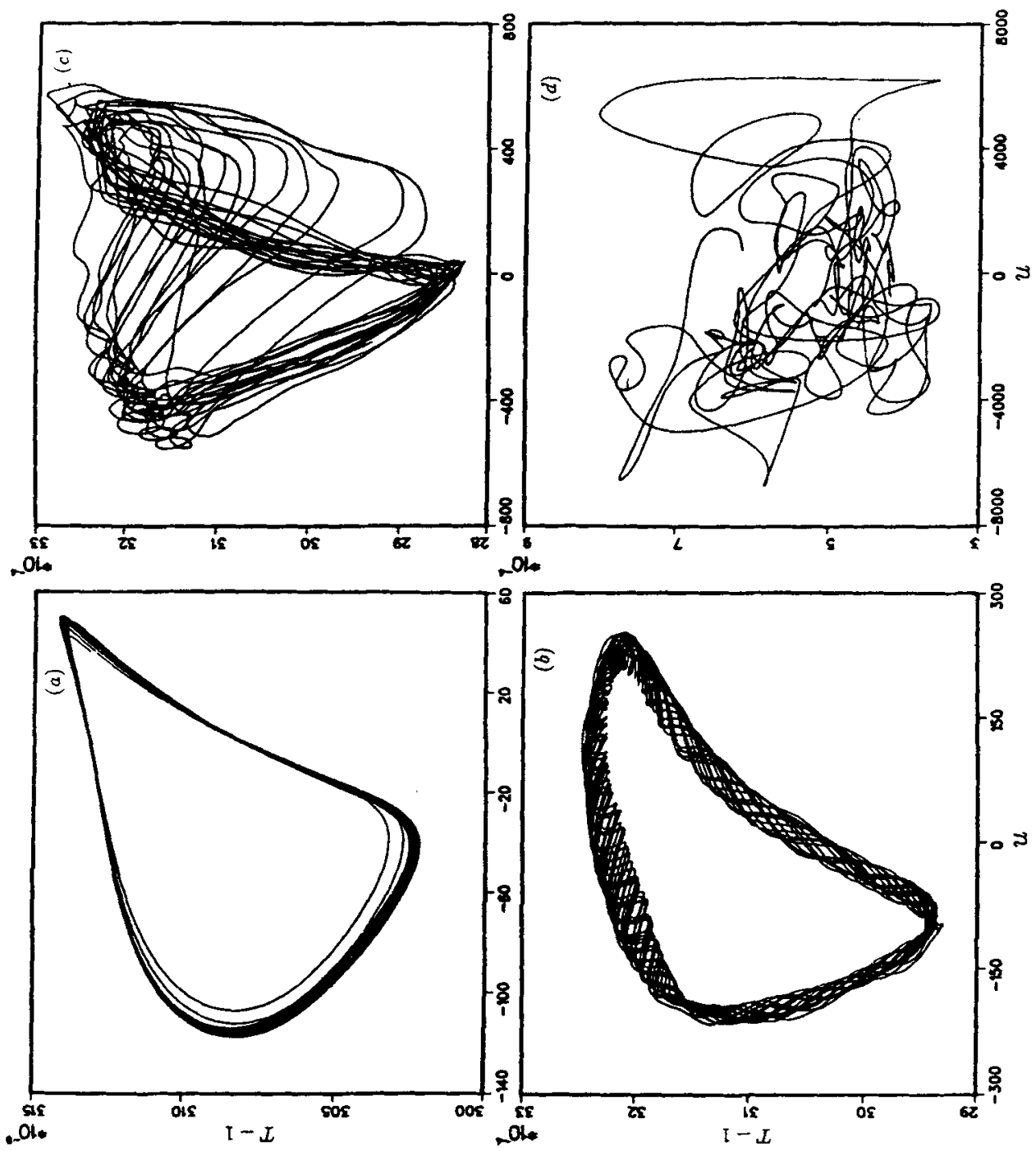
virtually immobile stably stratified fluid executing low-frequency, low-velocity oscillations. The temperature away from the boundary layers increases linearly over a large part of the height of the layer.

One of the earliest experimental studies of this problem was performed by Mull and Reiher and has been discussed by Jakob (1949) and Batchelor (1954). Subsequently Mordchelles-Regnier \& Kaplan (1963, pp. 94-111) have obtained some measurements and visualizations up to Rayleigh numbers of order $10^{12}$. These early works were followed by the classic experimental work of Elder (1965b), who measured primarily the temperature and velocity in the cavity using several fluids of high Prandtl number. He concluded that the occurrence of the turbulent wall layers in the cavity is independent of the aspect ratio and occurs in a similar manner to that on an isolated vertical plate. Subsequently, MacGregor \& Emery (1969) experimentally obtained heat-transfer data for different Prandtl numbers and aspect ratios; Kutateladze et al $(1972 \mathrm{a}, 1977,1978)$ measured heat transfer, velocity and temperature means, their fluctuations, as well as the probability density distribution of temperature fluctuations; Cowan et al (1982, pp. 195-203) obtained overall and local heat-transfer data for different aspect ratios; Kirdyashkin et al (1983) and Kirdyashkin \& Semenov (1984) measured the temperature means, fluctuations and their frequency spectra, the temperature kurtosis and skewness parameters, as well as other turbulence quantities; and Giel \& Schmidt (1986, pp. 1459-64) obtained velocity and temperature means and fluctuations, as well as frequency spectra of temperature fluctuations.

Eckert \& Carlson (1961), and Kutateladze et al (1972b, 1977) noted that the inner structure of the thermal boundary layer is locally similar to that of an isolated plate. More recently George \& Capp (1979), using classical scaling arguments, presented a theory for turbulent natural-convection boundary layers next to heated vertical surfaces. They showed that the boundary layer must be treated in two parts: an outer region, in which the viscous and conduction effects are negligible, and an inner region, in which the mean convection terms are negligible. The inner layer, which is identified as a constant heat-flux layer in the sense that the total heat flux across the layer is independent of the distance from the wall, consists of two regions: a conductive and viscous sublayer, in which the temperature is linear and the velocity approximately linear next to the wall, and a buoyant sublayer outside of it, where the mean velocity and temperature profiles depend on the cube root and inverse cube root of distance from the wall, respectively.

Paolucci (1990) considers the nonlinear physics of turbulence numerically. More specifically, he performs a direct numerical simulation of the free convective flow in the cavity for $A_{H}=1, \varepsilon \rightarrow 0, \operatorname{Pr}=0.71$, and $\mathrm{Ra}=10^{10}$. Although the initial conditions he used are non-random (quiescent and isothermal fluid), due to strong buoyancy the flow breaks up into an apparently random one. In general a flow which exhibits temporal as well as spatial randomness is identified as being turbulent.

The flow is again assumed to be two-dimensional. Although physical flows are three-dimensional, two-dimensional turbulence is often considered as a first approximation in many cases (e.g. turbulent flows submitted to a stable stratification). Furthermore, it appears from numerous experiments dealing with this problem (e.g., Giel \& Schmidt 1986, pp. 1459-64), and the related problem of natural convection from a heated vertical plate (e.g., Lochet et al 1983), that three-dimensionality in this class of flows has a very small influence if the depth dimension is not too small. In this connection, it is noted that the inertial sub-range of two-dimensional turbulence is quite different from that in three-dimensions (Kraichnan 1967; Lilly 1969). In 
particular, in inviscid isothermal flow the cascade of energy in two dimensions is mainly toward low wave number in the inertial range, and becomes weak or nonexistent at higher wave numbers where only vorticity variance or enstrophy is cascaded in the usual sense in the inertial range.

Paolucci's (1990) results show that convection flow in a vertical layer for Rayleigh numbers in the turbulent regime passes through several stages. In the lower part of the cavity, next to the heated wall, the boundary layer flow has laminar character. This region is followed by transition and finally by a turbulent boundary layer. The space between the vertical boundary layers is filled by a virtually immobile stably stratified fluid executing low-frequency, low-velocity oscillations. This central core is continually stirred by random buoyant elements of finite energy which are discharged into it.

In accord with the picture of George \& Capp (1979), the simulation shows that the thermal and momentum boundary layers can each be characterized by three regions. Directly against the wall there are the conductive and viscous sublayers, where the heat flux is constant but the shear stress is not. Defining the time averaged inner-region temperature and vertical velocity at the mid-height of the cavity $\left(y=A_{H} / 2\right)$ as

$$
\theta=\left(\bar{T}-\bar{T}_{m}\right) /\left(T_{h}-\bar{T}_{m}\right), \quad V_{\eta}=\eta \bar{v},
$$

where $\eta=\left(\beta_{r} g \Delta T_{m} L^{3} / \alpha_{r}^{2}\right)^{-1 / 3}$ is a dimensionless thermal inner scale, $\bar{T}_{m}=\bar{T}(1 / 2, y)$, and $\Delta T_{m}=T_{h}-T_{m}$, these regions are then given by

and

$$
\theta=1-0 \cdot 118(x / \eta) \text { for } 0 \leqslant x / \eta<3 \cdot 3 \text {, }
$$

$$
V_{\eta}=\operatorname{Pr}^{-1}\left[3 \cdot 89(x / \eta)-\frac{1}{2}(x / \eta)^{2}+(0 \cdot 118 / 6)(x / \eta)^{3}\right], \text { for } 0 \leqslant x / \eta<1 \cdot 5 \text {. }
$$

In these regions a monotonic rise in the intensity of fluctuations is seen. These regions are followed by the thermal and momentum buoyant sublayers whose variations with distance from the wall are given by

and

$$
\theta=2.406(x / \eta)^{-1 / 3}-0.943, \text { for } 3.3<x / \eta<13 \text {, }
$$

$$
V_{\eta}=13.63(x / \eta)^{1 / 3}-8 \cdot 77, \text { for } 1.5<x / \eta<3.3
$$

Thermal energy transferred by conduction accumulates in the thermal sublayer giving rise to fluctuations which constitute a considerable part of the heat transfer. Note that for $\operatorname{Pr}=0.71$ the momentum buoyant sublayer is fully within the conductive sublayer. Exterior to the mean vertical velocity maximum the simulation results shows that the velocity distribution is self-similar and given by

$$
\left.\bar{v} / \bar{v}_{m}=\exp \left[-0.947\left[\left(x-x_{m}\right) / \delta_{\bar{v}}\right)\right]^{1.432}\right] \text {, }
$$

where $\bar{v}_{m}=2.159 \times 10^{4}$ and $x_{m}=4.649 \times 10^{-3}$ are the maximum vertical velocity at the mid-height and the location away from the hot wall at which the maximum is found, and $\delta_{\bar{v}}=\int_{0}^{1 / 2}\left(\bar{v} / \bar{v}_{m}\right) \mathrm{d} x=1.214 \times 10^{-2}$ is the momentum boundary layer thickness. In this strong mixing region, wave-like structures are superimposed on the mean motion. As elements of these structures accelerate out of the region, a local reduction of the thermal energy is observed. The elements move sufficiently rapidly 
out of this highly intermittent region for molecular processes to be negligible. The external part of the thermal boundary layer is characterized by a small value of excess mean temperature.

It is seen that the stable stratification of the fluid outside the boundary layers significantly affects the nature of the flow, but has no effect on the heat transfer and momentum in the conductive and viscous sublayers. In the core of the cavity it is found that the temperature is approximately linearly stratified with a slope of

$$
S=\left(A_{H} / 2 \varepsilon\right)(\mathrm{d} \bar{T} / \mathrm{d} y)=0 \cdot 38
$$

This result appears to be independent of $A_{H}, \mathrm{Pr}$, and most surprisingly $\mathrm{Ra}$ since it is in good agreement with the experimental results obtained over a wide range of Rayleigh numbers by Elder (1965) who obtained $S=0.3-0.4$, for $10<A_{H}<30$, and $\operatorname{Pr}=7$, Kutateladze et al (1972a) who obtained $S=0.33$ for $A_{H}=22$ and $\operatorname{Pr}=13.2$, and Kutateladze et al $(1977,1978)$ who obtained $S=0.36 \pm 0.04$ for $8<A_{H}<26$ and $\operatorname{Pr} \approx 16$. In addition, using the statistically steady mean temperature gradient and averaging over the height of the cavity, he showed that the average Nusselt number can be written as

$$
\mathrm{Nu}=0.046 \mathrm{Ra}^{1 / 3}
$$

The agreement with the experimental values of Elder (1965b), MacGregor \& Emery (1969), Kutateladze et al (1977), Cowan et al (1982), and Kirdyashkin et al (1983), is excellent, and, as with the value of $S$ in (37), the constant 0.046 appears to be independent of the Prandtl number and aspect ratio, since the experimental results were obtained for $1 \leqslant \operatorname{Pr} \leqslant 10^{3}$ over the aspect ratio range $1<A_{H}<61$.

In comparing his predictions with available experimental evidence, many of Paolucci's (1990) results could be substantiated either directly or upon replotting data available in the literature. In particular, the heat-transfer law, the vertical stratification in the core, the viseous and conducting sublayers, the existence of momentum and thermal buoyant sublayers, and the self-similar distribution of the outer boundary layer region are in good quantitative agreement with available data. Many of the features observed in the flow such as the sinusoidal mode of instability, the internal jumps in the departing corners, and the "hook" like structures have been observed experimentally. However, the validity of many other results can only be verified when more experimental data become available.

Two important points can be made from the direct simulation results. First, there is now strong evidence that the prevailing theories of heat and mass transfer which assume relationships for friction and heat transfer, similar to those in forced boundary layer flow, are not applicable to natural-convection turbulence. Second, the results should be helpful in constructing simplified turbulence models for natural-convection flows in the future.

\section{Conclusions}

We have presented a brief review of recent work on the natural convection flow in enclosures. The prototypical configuration used in studying these flows is that of a vertical rectangular enclosure with lateral heating. The buoyancy forces in this review are considered to result exclusively from temperature differences applied across two 
vertical walls. The natural convection flows discussed have been primarily in enclosures in which $A_{D} \rightarrow \infty$, and for both large and small vertical aspect ratios $A_{H}$.

It is apparent from our discussion that the subject of natural convection in twodimensional large and small vertical aspect ratios and in the Boussinesq limit has a rather long history. Most of the elementary aspects of these flows in the laminar regime are now fairly well understood. More research is needed in the following areas.

(1) Laminar and transitional flows in cavities where $A_{H}=O(1)$, and $A_{D}=O(1)$ in both the Boussinesq and non-Boussinesq regimes. Very limited work has been done in this area computationally by Mallinson \& De Vahl Davis (1977) and Fusegi et al (1991), and experimentally by N'Dame (1992) who recently gave evidence of a possible low-frequency three-dimensional instability for $A_{D} \geqslant 1.37$.

(2) More complete study of the multiple solution regimes first found by Briggs \& Jones (1985). Some of this work has been started by Briggs \& Jones (1989), Le Quéré \& Alziary de Roquefort (1988), Le Quéré (1990), and Penot et al (1990, pp. 417-22).

(3) A more accurate and complete study of natural convection turbulence. This should be done using a more accurate method than that used by Paolucci (1990) to verify many of his results, should be extended to three-dimensions to eliminate many of the questions that have been raised about that work, and should be further extended to the full non-Boussinesq regime. In addition, and most importantly, these direct simulation results should be used to generate simple models to replace current models that are fundamentally based on high Reynolds number forced convection theories that assume classical relationships between friction and heat transfer.

\section{References}

Al-Homoud A A, Bejan A 1979 Experimental study of high Rayleigh number convection in a horizontal cavity with different end temperatures. Tech Rep. CUMER-79-1, University of Colorado, Boulder, Dept. of Mech. Eng.

Arakawa A 1966 Computational designs for long term numerical integration of the equations of fluid motion - Part 1. Two dimensional incompressible flow. J. Comput. Phys. 1:119-143

Batchelor G K 1954 Heat transfer by free convection across a closed cavity between vertical boundaries at different temperatures. Q. Appl. Math. 12: 209-233

Bejan A 1979 Note on Gill's solution for free convection in vertical enclosure. J. Fluid Mech. 90: $561-568$

Bejan A 1980 A synthesis of analytical results for natural convection heat transfer across rectangular enclosures. Int. J. Heat Mass Transfer 23: 723-726

Bejan A,. Al-Homoud A A, Imberger J 1981 Experimental study of high Rayleigh number convection in a horizontal cavity with different end temperatures. J. Fluid Mech. 109: 283-299

Bejan A, Tien C L 1978 Laminar natural convection heat transfer in a horizontal cavity with different end temperatures. J. Heat Transfer 100: 641-647

Bergholz R F 1978 Instability of steady natural convection in a vertical fluid layer. J. Fluid Mech. 84: 743-768

Briggs D G, Jones D N 1985 Two-dimensional periodic natural convection in a rectangular enclosure of aspect ratio one. J. Heat Transfer 107: 850-854

Briggs D G, Jones D N 1989 Periodic two-dimensional cavity flow: effect of linear horizontal thermal boundary conditions. J. Heat Transfer 111: 86-91

Catton I 1978 Natural convection in enclosures. In Heat Transfer 1978 (Washington, DC: Hemisphere) vol. 6 
Chenoweth D R, Paolucci S 1985 Gas flow in vertical slots with large horizontal temperature differences. Phys. Fluids 28: 2365-2374

Chenoweth D R, Paolucci S 1986 Natural convection in an enclosed vertical air layer with large horizontal temperature differences. J. Fluid Mech. 169: 173-210

Cormack D E, Leal L G, Imberger J 1974a Natural convection in a shallow cavity with differentially heated end walls. Pt. 1, Asymptotic theory. J. Fluid Mech. 65: 209-229

Cormack D E, Leal L G, Seinfield J H 1974b Natural convection in a shallow cavity with differentially heated end walls. Pt. 2, Numerical solutions. J. Fluid Mech. 65: 231-246

Cowan G H, Lovegrove P C, Quarini G L 1982 Turbulent natural convection heat transfer in vertical single water-filled cavities. In Heat Transfer 1982 (eds) U Grigull, E Hahne, K Stephan, J Straub (Washington DC: Hemisphere) vol. 2

De Vahl Davis G, Jones I P 1983 Natural convection in a square cavity: a comparison exercise. Int. J. Numer. Meth. Fluids 3: 227-248

Deardorff J W 1973 Three-dimensional numerical modelling of the planetary boundary layers. In Workshop on micrometeorology (Washington, DC: Am. Math. Soc.)

Drummond J E, Korpela S A 1987 Natural convection in a shallow cavity. J. Fluid Mech. 182: $543-564$

Duxbury D 1979 An interferometric study of natural convection in enclosed plane air layers with complete and partial central vertical divisions. Ph D thesis, University of Salford, UK

Eckert E R G, Carlson W O 1961 Natural convection in an air layer enclosed between two vertical plates with different temperatures. Int. J. Heat Mass Transfer 2: 106-120

Elder J W 1965a Laminar free convection in a vertical slot. J. Fluid Mech. 23: 77-98

Elder J W 1965b Turbulent free convection in a vertical slot. J. Fluid Mech. 23: 99-111

Fenstermacher P R, Swinney H L, Gollub J P 1979 Dynamical instabilities and the transition to chaotic Taylor vortex flow. J. Fluid Mech. 94: 103-128

Fusegi T, Hyun J M, Kuwahara K, Farouk B 1991 A numerical study of three-dimensional natural convection in a differentially heated cubical enclosure. Int. J. Heat Mass Transfer 34: $1543-1557$

George W K, Capp S P 1979 A theory for natural convection turbulent boundary layers next to heated vertical surfaces. Int. J. Heat Mass Transfer 22: 813-826

Giel P W, Schmidt F W 1986 An experimental study of high Rayleigh number natural convection in an enclosure. In Heat Transfer 1986 (eds) C L Tien, V P Carey, J K Ferrell (Washington: DC: Hemisphere) vol. 4

Gill A E 1966 The boundary-layer regime for convection in a rectangular cavity. J. Fluid Mech. 26: 515-536

Gill A E, Davey A 1969 Instabilities of a buoyancy-driven system. J. Fluid Mech. 35: 775-798

Gollub J P, Benson S V 1980 Many routes to turbulent convection. J. Fluid Mech. 100:449-470

Gorman M. Swinney H L 1982 Spatial and temporal characteristics of modulated waves in the circular Couette system. J. Fluid Mech. 117: 123-142

Graebel W P 1981 The influence of Prandtl number on free convection in a rectangular cavity. Int. J. Heat Mass Transfer 23: 125-131

Gray D D, Giorgini A 1976 The validity of the Boussinesq approximation for liquids and gases. Int. J. Heat Mass Transfer 19: 545-551

Haldenwang P 1986 Unsteady numerical simulation by Chebyshev spectral methods of natural convection at high Rayleigh number. J. Fluid Mech. 144: 389-401

Hara T 1958 Heat transfer by laminar free convection about a vertical flat plate with large temperature difference. Bull. JSME 1: 251-254

Hart J E 1972 Stability of thin non-rotating Hadley circulations. J. Atmos. Sci. 29: 687-697

Hart J E 1983 Low Prandtl number convection between differentially heated end walls. Int. J. Heat Mass Transfer 26: 1069-1074

Imberger J 1974 Natural convection in a shallow cavity with differentially heated end walls. Pt. 3, Experimental results. J. Fluid Mech. 65: 247-260

Ivey G N 1984 Experiments on transient natural convection in a cavity. J. Fluid Mech. 144: 389-401

Iyer P A 1973 Instabilities in buoyancy-driven boundary-layer flows in a stably stratified medium. Boundary-Layer Meteorology 5: 53-66

Jakob M 1949 Heat transfer (New York: Wiley)

Keunecke V K -H 1970 Stehende interne Wellen in rechteckigen Becken (Standing internal waves in rectangular tanks). Deutsche Hydrographische Zeitschrift 23: 61-79 
Kirdyashkin A G, Semenov V I 1984 Spectra of temperature fluctuations in a vertical layer with thermogravitational convection. High Temperature 21: 558-565

Kirdyashkin A G, Semenov V I, Berdnikov V S, Gaponov V A 1983 Structure of the temperature field in a vertical layer with thermal gravitational convection. High Temperature 20:750-757

Korpela S A 1974 A study of the effect of Prandtl number on the stability of the conduction regime of natural convection in an inclined slot. Int. J. Heat Mass Transfer 17: 215-222

Korpela S A, Gözüm D, Baxi C B 1973 On the stability of the conduction regime of natural convection in a vertical slot. Int. J. Heat Mass Transfer 16: 1683-1690

Kraichnan R H 1967 Inertial ranges in two-dimensional turbulence. J. Phys. Fluids 10: $1417-1423$

Kutateladze S S, Ivakin V P, Kirdyashkin A G, Kekalov A N 1977 Turbulent natural convection in a vertical layer. High Temperature 15: 458-464

Kutateladze S S, Ivakin V P, Kirdyashkin A G, Kekalov A N 1978 Thermal free convection in a liquid in a vertical slot under turbulent flow conditions. Heat Transfer-Soviet Res. 10: $118-125$

Kutateladze S S, Kirdyashkin A G, Ivakin V P 1972a Turbulent natural convection on a vertical plate and in a vertical layer. Int. J. Heat Mass Transfer 15: 193-202

Kutateladze S S, Kirdyashkin A G, Ivakin V P 1972b Turbulent natural convection at an isothermal vertical plate. High Temperature 10: 76-79

Lankhorst A M, Hoogendoorn C J 1988 Three-dimensional numerical calculations of high Rayleigh number natural convective flows in enclosed cavities. In Proc. 1988 National Heat Transfer Conf., ASME HTD-96 (New York: ASME) 3: 463-470

Lauriat G 1980 Numerical study of natural convection in a narrow vertical cavity: an examination of high-order accuracy schemes. ASME Paper 80-HT-90

Le Quéré P 1990 A note on multiple and unsteady solutions in two-dimensional convection in a tall cavity. J. Heat Transfer 112: 965-974

Le Quéré P, Alziary de Roquefort T 1985 Computation of natural convection in twodimensional cavities with Chebyshev polynomials. J. Comput. Phys. 57: 210-228

Le Quéré P, Alziary de Roquefort T 1986 Transition to unsteady natural convection in a differentially heated cavity In Heat Transfer 1986 (eds) C L Tien, V P Carey, J K Ferrell (Washington, DC: Hemisphere) vol. 4

Le Quéré P, Alziary de Roquefort T 1988 Sur l' existence de solutions périodiques multiples aux équations de Boussinesq. C. R. Acad. Sci. Paris, II 306: 681-687

Le Quéré P, Masson R, Perrot P 1992 A Chebyshev collocation algorithm for 2D nonBoussinesq convection. J. Comput. Phys. 103: 320-335

Lee T S, Son G H, Lee J S 1988 Numerical predictions of three-dimensional natural convection in a box. In Proc. 1st KSME-JSME Thermal and Fluids Eng. Conf. 2: 278-283

Lee Y, Korpela S A, 1983 Multicellular natural convection in a vertical slot. J. Fluid Mech. 126: $91-121$

Leonardi E, Reizes J A 1979 Natural convection in compressible fluids with variable properties. In Numerical methods in thermal problems (eds) $\mathbf{R}$ W Lewis, $\mathbf{K}$ Morgan (Swansea, UK: Pineridge)

Leonardi E, Reizes J A 1981 Convective flows in closed cavities with variable fluid properties. In Numerical methods in heat transfer (eds) R W Lewis, K Morgan, O C Zienkiewicz ( New York: John Wiley)

Lilly D K 1969 Numerical simulation of two-dimensional turbulence. Phys. Fluids Supp. II 12: $240-249$

Lochet R, Lemonnier D, Doan-Kim-Son 1983 Correlations en convection naturelle turbulente influence de la pression et de la nature du gaz. Int. J. Heat Mass Transfer 26: 1221-1227

MacGregor R K, Emery A F 1969 Free convection through vertical layers-Moderate and high Prandtl number fluids. J. Heat Transfer 91: 391-401

Mallinson G D, De Vahl Davis G 1977 Three-dimensional natural convection in a box: a numerical study. J. Fluid Mech. 83: 1-31

Mordchelles-Regnier G, Kaplan C 1963 Visualization of natural convection on a plane wall and in a vertical gap by differential interferometry: transitional and turbulent regimes. In Heat Transfer and Fluid Mechanics Institute (Stanford: University Press)

N'Dame A 1992 Etude expérimentale de la convection naturelle en cavité: de l'état stationnaire au chaos. Ph D thesis, Université de Poitiers, France 
Newhouse S, Ruelle D, Takens P 1978 Occurrence of strange axiom A attractors near quasi periodic flows on $T^{m}, m \geqslant 3$. Commun. Math. Phys. 64: 35-40

Ostrach S 1972 Natural convection in enclosures. In Advances in heat transfer (New York: Academic Press) vol. 8

Ostrach S 1982 Natural convection heat transfer in cavities and cells. In Heat Transfer 1982 (ed.) U Grigull, E Hahne, K Stephan, J Straub (Washington, DC: Hemisphere) vol. 1

Paolucci S 1982 On the filtering of sound from the Navier-Stokes equations. Tech. Rep. SAND82-8257, Sandia National Laboratories Report

Paolucci S 1990 Direct numerical simulation of two-dimensional turbulent natural convection in an enclosed cavity. J. Fluid Mech. 215: 229-262

Paolucci S, Chenoweth D R 1988 Natural convection in shallow enclosures with differentially heated endwalls. J. Heat Transfer 110: 625-634

Paolucci S, Chenoweth D R 1989 Transition to chaos in a differentially heated vertical cavity. J. Fluid Mech. 201: 379-410

Patterson J C 1984 On the existence of an oscillatory approach to steady natural convection in cavities. J. Heat Transfer 106: 104-108

Patterson J C, Imberger J 1980 Unsteady natural convection in a rectangular cavity. J. Fluid Mech. 100: 65-86

Penot F, N'Dame A, Le Quéré P 1990 Investigation of the route to turbulence in a differentially heated cavity. In Heat Transfer 1990 (ed.) G Hetsroni (Washington, DC: Hemisphere) vol. 2

Peyret R, Taylor T D 1983 Computational methods for fluid flow (New York: Springer)

Piacsek S A, Williams G P 1970 Conservation properties of convection difference schemes. $J$. Comput. Phys. 6: 362-392

Polezhaev VI 1967 Numerical solution of a system of two-dimensional unsteady Navier-Stokes equations for a compressible gas in a closed region. Fluid Dynamics 2: 70-74

Polezhaev V I 1968 Flow and heat transfer in laminar natural convection in a vertical layer. Teplo i Massoobmen 1: 631-640

Quon C 1972 High Rayleigh number convection in an enclosure - A numerical study. Phys. Fluids 15: 12-19

Roux B, Grondin J C, Bontoux P, De Vahl Davis G 1980 Reverse transition from multicellular to monocellular motion in vertical fluid layer. Phys. Chem. Hydrodyn. 3: 292-297

Rubel A, Landis F 1970 Laminar natural convection in a rectangular enclosure with moderately large temperature differences. In Heat Transfer 1970 (eds) U Grigull, E Hahne (Washington, DC: Hemisphere) vol. 4

Shiralkar G S, Gadgil A, Tien C L 1981 High Rayleigh number convection in shallow enclosures with different end temperatures. Int. J. Heat Mass Transfer 24: 1621-1629

Sparrow E M, Gregg J L 1958 The variable fluid-property problem in free convection. Trans. ASME 80: 879-886

Thorpe S A 1968 On standing internal gravity waves of finite amplitude. J. Fluid Mech. 32: 489-528

Tichy J, Gadgil A 1982 High Rayleigh number laminar convection in low aspect ratio enclosures with adiabatic horizontal walls and differentially heated vertical walls. $J$. Heat Transfer 104: $103-110$

Vest C M, Arpaci V S 1969 Stability of natural convection in a vertical slot. J. Fluid Mech. 36: $1-15$

White F M 1974 Viscous fluid flow (New York: McGraw-Hill)

Yang K T 1987 Natural convection in enclosures. In Handbook of single-phase convective heat transfer (eds) S Kakac, R K Shah, W Aung (New York: Wiley-Interscience)

Yang K T 1988 Transitions and bifurcations in laminar buoyant flows in confined enclosures. J. Heat Transfer 110: 1191-1204

Yewell R, Poulikakos D, Bejan A 1982 Transient natural convection - Experiments in shallow enclosures. J. Heat Transfer 104: 533-538

Yin S H, Wung T Y, Chen K 1978 Natural convection in an air layer enclosed within rectangular cavities. Int. J. Heat Mass Transfer 21: 307-315 